\title{
Vigilant: An engineered VirD2-Cas9 complex for lateral flow assay-based detection of SARS-CoV2
}

Tin Marsic ${ }^{1}$, Zahir Ali ${ }^{1}$, Muhammad Tehseen ${ }^{2}$, Ahmed Mahas ${ }^{1}$, Samir Hamdan ${ }^{2}$, and Magdy Mahfouz $^{1, *}$

${ }^{1}$ Laboratory for Genome Engineering and Synthetic Biology, Division of Biological Sciences, 4700 King Abdullah University of Science and Technology, Thuwal 23955-6900, Saudi Arabia. ${ }^{2}$ Laboratory of DNA Replication and Recombination, Biological and Environmental Sciences and Engineering Division, King Abdullah University of Science and Technology (KAUST), Thuwal 23955-6900, Saudi Arabia.

*Correspondence: Magdy M. Mahfouz (magdy.mahfouz@kaust.edu.sa)

Key words: COVID-19, SARS-CoV-2, RT-RPA, lateral flow assay, CRISPR-Cas9, nucleic acid detection, VirD2, dCas9, biosensors, molecular diagnostics, relaxases 


\section{MATERIALS AND METHODS}

\section{Nucleic acid preparation}

\section{a) Plasmids, ssDNA probe, and oligos}

VirD2-SpCas9 and SpCas9-VirD2 clones were used to purify the fusion proteins. Mutant VirD2SpdCas9 (dead SpCas9-VirD2), SpdCas9-VirD2 (VirD2-dead SpCas9), and SpdCas9-dVirD2 (dead SpCas9-dead VirD2) clones for expression of the respective fusion proteins were custom synthesized by GenScript. Guide RNAs for SpCas9 experiments were designed using SnapGene and ordered as gBlocks from Integrated DNA Technologies under the T7 promoter for in vitro transcription. The FAM-labeled ssDNA probe and biotin-labeled oligos were ordered from Integrated DNA Technologies. Sequences of the plasmids and oligos are listed in the supplementary file.

\section{b) In vitro transcription of $\mathrm{SgRNA}$}

In vitro transcription was performed using TranscriptAid T7 High Yield Transcription Kit (Thermo Scientific) following the manufacturer's instructions. Briefly, $10 \mu \mathrm{L}$ of $5 \mathrm{X}$ TranscriptAid Reaction Buffer, $20 \mu \mathrm{L}$ NTP mix, $10 \mu \mathrm{L}$ of the DNA template (annealed sgRNA gBlock and T7 promoter oligo), $0.5 \mu \mathrm{L}$ of RNase Out, $5 \mu \mathrm{L}$ of TranscriptAid Enzyme Mix, and $4.5 \mu \mathrm{L}$ of DEPC-treated water were incubated at $37^{\circ} \mathrm{C}$ for 8 hours. In vitro transcribed RNA was purified using Direct-zol RNA MiniPrep Kit (Zymo Research). Production of the proper size sgRNA fragments was confirmed on a $2 \%$ agarose gel run in Tris-Borate-EDTA buffer.

\section{Protein purification}

Protein purification was performed as previously described [13]. Briefly, a single colony of BL21(DE3) was grown in 2X-YT media and induced at $0.6 \mathrm{OD}_{600}$ with $0.3 \mathrm{mM}$ IPTG and incubated at $18^{\circ} \mathrm{C}$ for $15 \mathrm{~h}$ at $180 \mathrm{rpm}$. Proteins were isolated using affinity purification column and further purified by size fractionation using the ÄKTA pure system (Cytiva).

\section{Functional characterization of in-house produced enzymes}




\section{a) SpCas9 nuclease activity assay of the fusion proteins}

Target fragment (SARS-CoV-2, $N$-gene fragment) was amplified by PCR for SpCas9-based cleavage assays. Ribo-nucleoprotein particles of VirD2-SpCas9, SpCas9-VirD2, and catalytically dead mutants VirD2-SpdCas9, SpdCas9-VirD2 and SpdCas9-dVirD2 were assembled at $37^{\circ} \mathrm{C}$ for 10 minutes in $16.3 \mu \mathrm{L}$ reaction consisting of $250 \mathrm{nM}$ of the respective protein, $250 \mathrm{nM}$ sgRNA in the cleavage buffer $(10 \mathrm{mM}$ Tris- $\mathrm{HCl} \mathrm{pH} 8.0,50 \mathrm{mM} \mathrm{NaCl}, 10 \mathrm{mM}$ $\mathrm{MgCl}_{2}$ ). Following the incubation, $3.7 \mu \mathrm{L}$ containing $150 \mathrm{ng}$ of the target was added into the tube and the reaction was incubated for 1 hour at $37^{\circ} \mathrm{C}$. The protein was denatured at $95^{\circ} \mathrm{C}$ for 5 minutes, the reaction was cooled on ice for 3 minutes, and the DNA products were separated on a $2 \%$ agarose gel.

\section{b) VirD2 and probe covalent binding assay of the fusion proteins}

Biotin-labeled probe (ssDNA harboring the T-DNA right border sequence) was mixed with the fusion proteins (VirD2-SpCas9, SpCas9-VirD2, and catalytically dead mutants VirD2-SpdCas9, SpdCas9-VirD2 and SpdCas9-dVirD2) at the ratio $1: 1$ (250 nM each) V2 buffer (10 mM Tris$\mathrm{HCl} \mathrm{pH} \mathrm{8.0,} 50 \mathrm{mM} \mathrm{NaCl}, 10 \mathrm{mM} \mathrm{MgCl}_{2}$ ). The reaction mix was incubated at $37^{\circ} \mathrm{C}$ for 60 minutes. Protein loading dye was added to the reaction and heated for $95^{\circ} \mathrm{C}$ for 3 minutes. The complex was resolved on $10 \% \mathrm{NuPAGE}$ (Invitrogen) for $3 \mathrm{~h}$ at $4{ }^{\circ} \mathrm{C}$ and transferred to nitrocellulose membrane for $3 \mathrm{~h}$ at $4^{\circ} \mathrm{C}$. The membrane was immuno-blotted with anti-biotin mouse primary antibody 1:1000 (Cat. No. SC-53179, Santa Cruz) and anti-mouse secondary antibody 1:2000 (Cat. No. A3688, Sigma) and detected with chemiluminescent ECL solution (BioRad). Alternatively, for direct gel mobility shift, probe (64 bp ssDNA harboring the T-DNA right border sequence) was mixed with the fusion proteins (VirD2-SpCas9, SpCas9-VirD2, and catalytically dead mutants VirD2-SpdCas9, SpdCas9-VirD2 and SpdCas9-dVirD2) at the ratio 1 : 1 (250 nM each) V2 buffer (10 mM Tris- $\mathrm{HCl} \mathrm{pH}=8.0,50 \mathrm{mM} \mathrm{NaCl}, 10 \mathrm{mM} \mathrm{MgCl}$ ). The reaction mix was incubated at $37^{\circ} \mathrm{C}$ for 60 minutes. Protein loading dye was added to the reaction and heated for $95^{\circ} \mathrm{C}$ for 3 minutes. The complex was resolved on $10 \% \mathrm{NuPAGE}$ (Invitrogen) for $3 \mathrm{~h}$ at $4^{\circ} \mathrm{C}$. Gel was stained with Coomassie brilliant blue stain (MBP). Gel photos were taken using Gel Doc XR (BioRad). 


\section{RT-RPA reactions with synthetic targets}

RT-RPA was performed using the TwistAmp Basic kit following the manufacturer's instructions. Briefly, a well-mixed $47.5-\mu \mathrm{L}$ sample (1 $\mu \mathrm{l} \mathrm{RNA}$ template, $2.4 \mu \mathrm{L}$ of $10 \mu \mathrm{M}$ biotinlabeled forward and $2.4 \mu \mathrm{L}$ of unlabeled reverse primers, $29.5 \mu \mathrm{L}$ of Rehydration buffer, $0.5 \mu \mathrm{L}$ of SuperScript IV reverse transcriptase, $1 \mu \mathrm{L}$ of RNase $\mathrm{H}, 0.5 \mu \mathrm{L}$ of RNase Out, $10.2 \mu \mathrm{L} \mathrm{H}_{2} 0$ ) was added to the lyophilized RPA reaction components (TwistAmp Basic) and homogenized by pipetting. Magnesium acetate $(2.5 \mu \mathrm{L}$ of $280 \mathrm{mM})$ was added to each tube and mixed. The isothermal amplification was performed at $42^{\circ} \mathrm{C}$ for 25 minutes. To confirm the DNA isothermal amplification, $10 \mu \mathrm{L}$ of the reactions were purified using QIAquick PCR Purification Kit and separated on a $1.5 \%$ agarose gel.

\section{VirD2-SpdCas9 reporter complex assembly}

The reporter complex (final concentration of $250 \mathrm{nM}$ each VirD2-SpdCas9, sgRNA and FAMlabeled reporter oligo) was prepared by combining $1.25 \mu \mathrm{L}$ of $5 \mu \mathrm{M}$ VirD2-SpCas9 secondary stock, $1.25 \mu \mathrm{L}$ of $5 \mu \mathrm{M}$ sgRNA, $1.25 \mu \mathrm{L}$ of $5 \mu \mathrm{M}$ FAM-labeled reporter oligo, $5 \mu \mathrm{L}$ of $5 \mathrm{X}$ RPA reaction buffer $(50 \mathrm{mM}$ Tris- $\mathrm{HCl} \mathrm{pH} 8.0,500 \mathrm{mM} \mathrm{KCl}, 250 \mathrm{mM} \mathrm{NaCl}, 5 \mathrm{mM}$ DTT, $50 \mathrm{mM}$ $\mathrm{MgCl}_{2}, 250 \mathrm{mM}$ L-arginine, and $250 \mathrm{mM}$ L-glutamic acid) or $5 \mu \mathrm{L} 5 \mathrm{X}$ PCR reaction buffer (100 mM HEPES pH 8.0, $500 \mathrm{mM} \mathrm{KCl,} 250 \mathrm{mM} \mathrm{NaCl}, 5 \mathrm{mM}$ DTT, $25 \mathrm{mM} \mathrm{MgCl}$ ), $0.5 \mu \mathrm{L}$ of RNase Out and ultra-pure water to $20 \mu \mathrm{L}$. The reaction was incubated at $37^{\circ} \mathrm{C}$ for 60 minutes. Two reactions, $20 \mu \mathrm{L}$ each, were combined in a single tube to make $40 \mu \mathrm{L}$ preassembled reporter reaction.

\section{VirD2-SpdCas9 detection assay and lateral flow assay}

RT-RPA product $(5 \mu \mathrm{L})$ was mixed with $40 \mu \mathrm{L}$ of preassembled reporter. The $45-\mu \mathrm{L}$ reaction mix was incubated at $37^{\circ} \mathrm{C}$ for 10 minutes followed by 1 minute at $60^{\circ} \mathrm{C}$. Following the incubation, $55 \mu \mathrm{L}$ of the running buffer $(44.5 \mu \mathrm{L}$ of the HybriDetect Assay Buffer with $10.5 \mu \mathrm{L}$ of $10 \% \mathrm{BSA}$ ) was added directly into the reaction and mixed. Room temperature adjusted HybriDetect Dipsticks were placed into the tube containing the reaction mixture. Lateral flow strips were removed from the tube as soon as the control band appeared and the result was called 
within 10 minutes. Images of the strips were taken within 25 minutes after the beginning of the LFA.

\section{Limit of detection assay}

SARS-CoV-2 RNA (synthetic RNA from IDT) was diluted to final concentrations corresponding to $1,2.5,7.5,10,50$, and 100 copies/ $\mu \mathrm{L}$ or $50,125,375,500,2500$, and 5000 copies/reaction. The respective volume of RNA sample was added to the RT-RPA reactions. Nuclease-free water was used as the negative control. Following the RT-RPA reaction, $5 \mu \mathrm{L}$ of the product was transferred into the reaction containing the preassembled reporter complex and the samples were detected as previously described. The detection limit was considered as the concentration that could be successfully detected within 10 minutes of the LFA assay in all three replicates.

\section{Validation of the developed protocol with SARS-CoV-2 clinical sample}

RNA samples from SARS-CoV-2 RT-PCR positive (26 clinical samples) and negative (4 clinical samples) were used for evaluation of our Vigilant protocol. RNA (4 $\mu \mathrm{L})$ was added to the RTRPA reaction and $5 \mu \mathrm{L}$ of the amplified product was used for detection in the next step. 


\section{List of Supplementary Figures}

Supplementary Figure 1. VirD2 alone is capable of cleaving ssDNA containing the specific RB recognition sequence.

Supplementary Figure 2. Covalent binding of VirD2 and ssDNA probe.

Supplementary Figure 3. Covalent binding of VirD2 and ssDNA.

Supplementary Figure 4. Confirmation of VirD2-SpCas9 and SpCas9-VirD2 nuclease activity.

Supplementary Figure 5. Selection of the optimal RT-RPA primer set.

Supplementary Figure 6. Selection of the optimal reaction buffer.

Supplementary Figure 7. Evaluation of the Vigilante at $42{ }^{\circ} \mathrm{C}$.

Supplementary Figure 8. Comparison of VirD2-SpCas9 and VirD2-SpdCas9.

Supplementary Figure 9. Repeats 2 and 3 of LOD determination.

Supplementary Figure 10. Copy number determination by RT-qPCR for $\mathrm{Ct}$ value relevance in clinical samples.

Supplementary Figure 11. Reporter complex stability.

Supplementary Figure 12. Additional evaluated clinical samples and their original scan.

Supplementary table 1. Designation and the $\mathrm{Ct}$ value of the clinical samples used in this study. RT-qPCR was performed on Trizol based isolated RNA. 

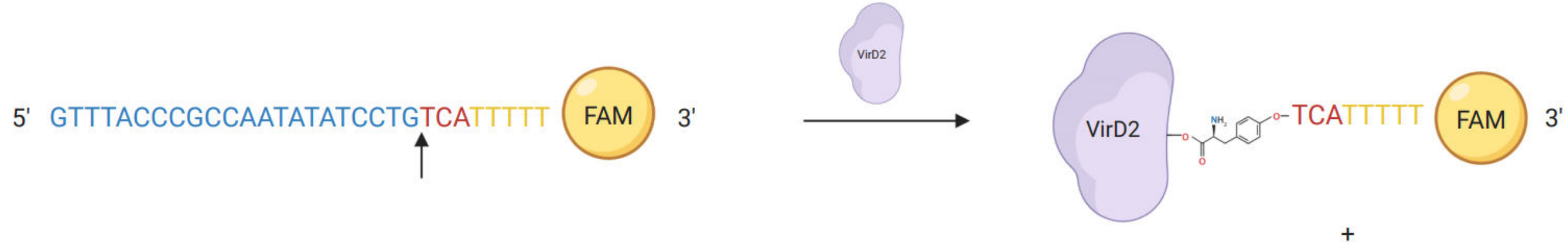

5' GTTTACCCGCCAATATATCCTG 3'

Supplementary Figure 1. VirD2 alone is capable of cleaving ssDNA containing the specific RB recognition sequence. Following the cleavage of the oligonucleotide, VirD2 remains covalently bound to Tyr29 moiety, leaving three nucleotides of the original sequence. This property can be exploited to attach labels at the $3^{\prime}$ end of oligonucleotide sequence bound to VirD2. 
A

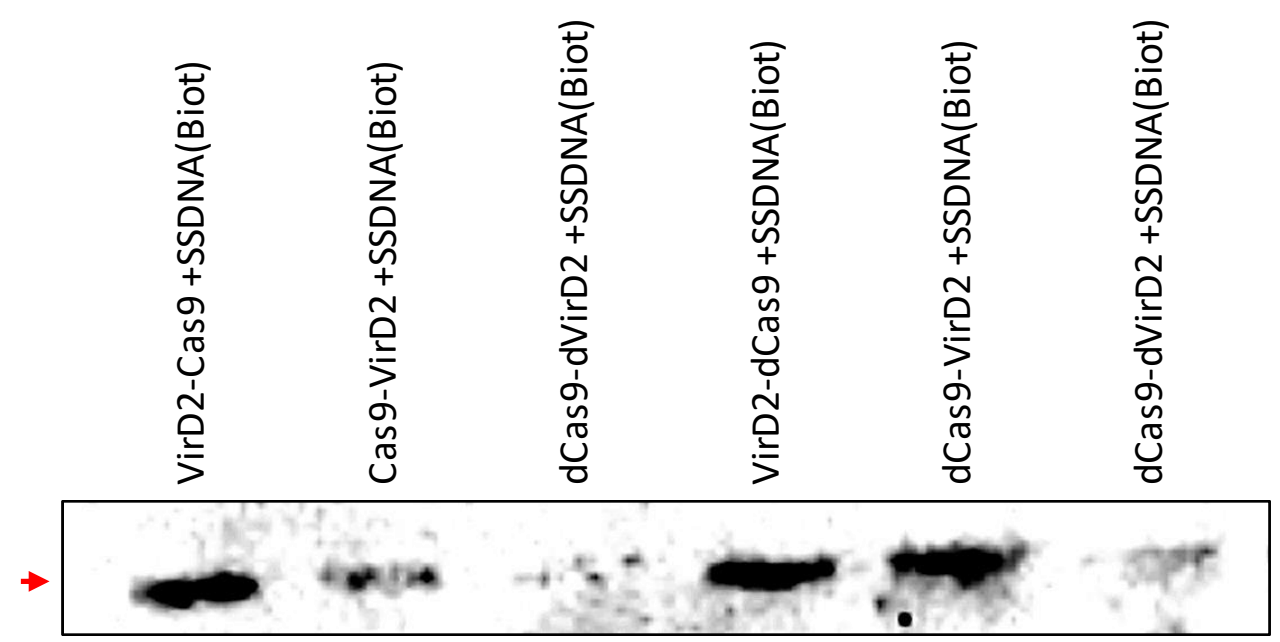

B

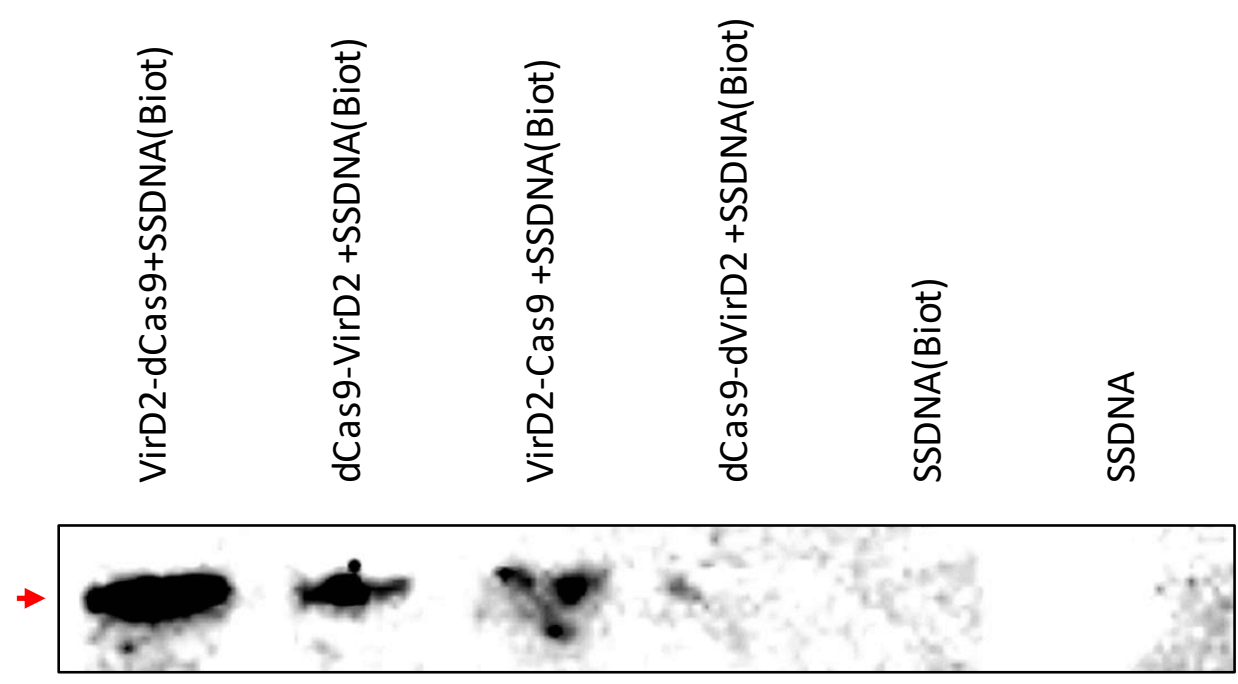

Supplementary Figure 2. A, B), Covalent binding of VirD2 and ssDNA probe. Biotin labeled probe harboring the T-DNA right border sequence was incubated with fusion proteins in the presence of $\mathrm{Mg}^{2+}$. VirD2-Cas9, Cas9-VirD2, VirD2-dCas9, dCas9-VirD2 bound to biotin labeled probe were detected by western blot. Biotin labeled probe, unlabeled probe, and dCas9-dVirD2 (no binding to RB sequence containing probe) were used as experimental control. Red arrow head indicated the immuno-detection of the biotin labeled probe bounded to fusion proteins. 
A

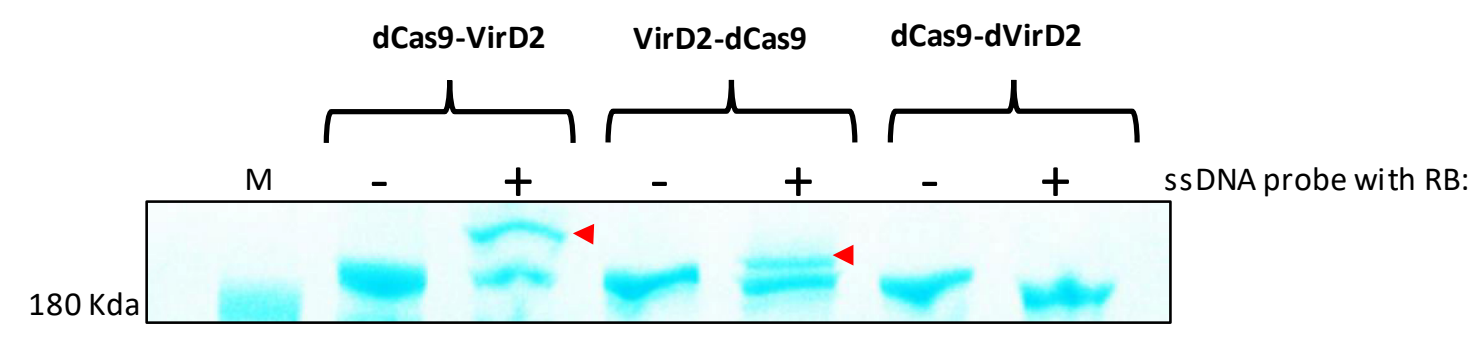

B

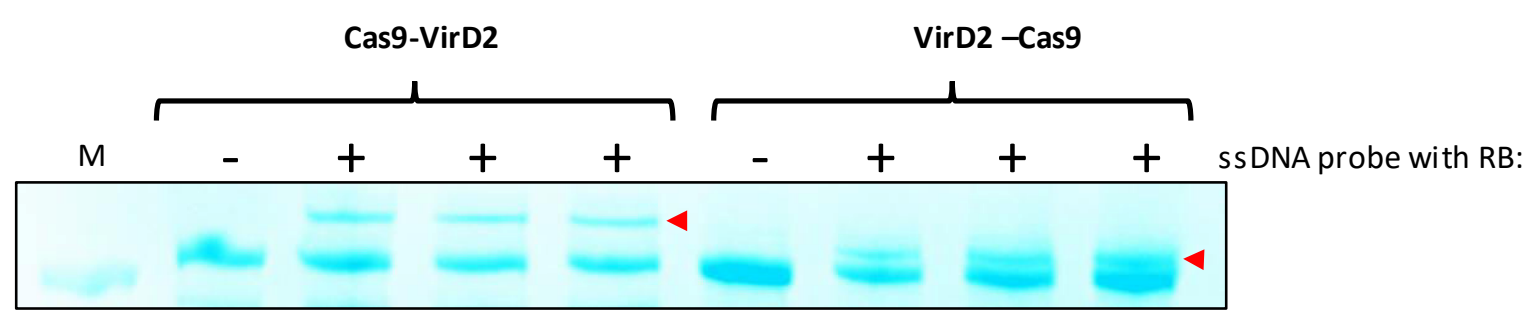

Supplementary Figure 3. Covalent binding of VirD2 and ssDNA. ssDNA strand (64 bp), harboring the T-DNA right border sequence was incubated with fusion proteins in the presence of $\mathrm{Mg}^{2+}$. VirD2-Cas9, Cas9-VirD2, VirD2-dCas9, dCas9-VirD2 bound to biotin labeled probe were detected by Coomassie staining. RB containing probe did not bind dCas9-dVirD2. Red arrow head indicated the ssDNA - fusion protein complex. 


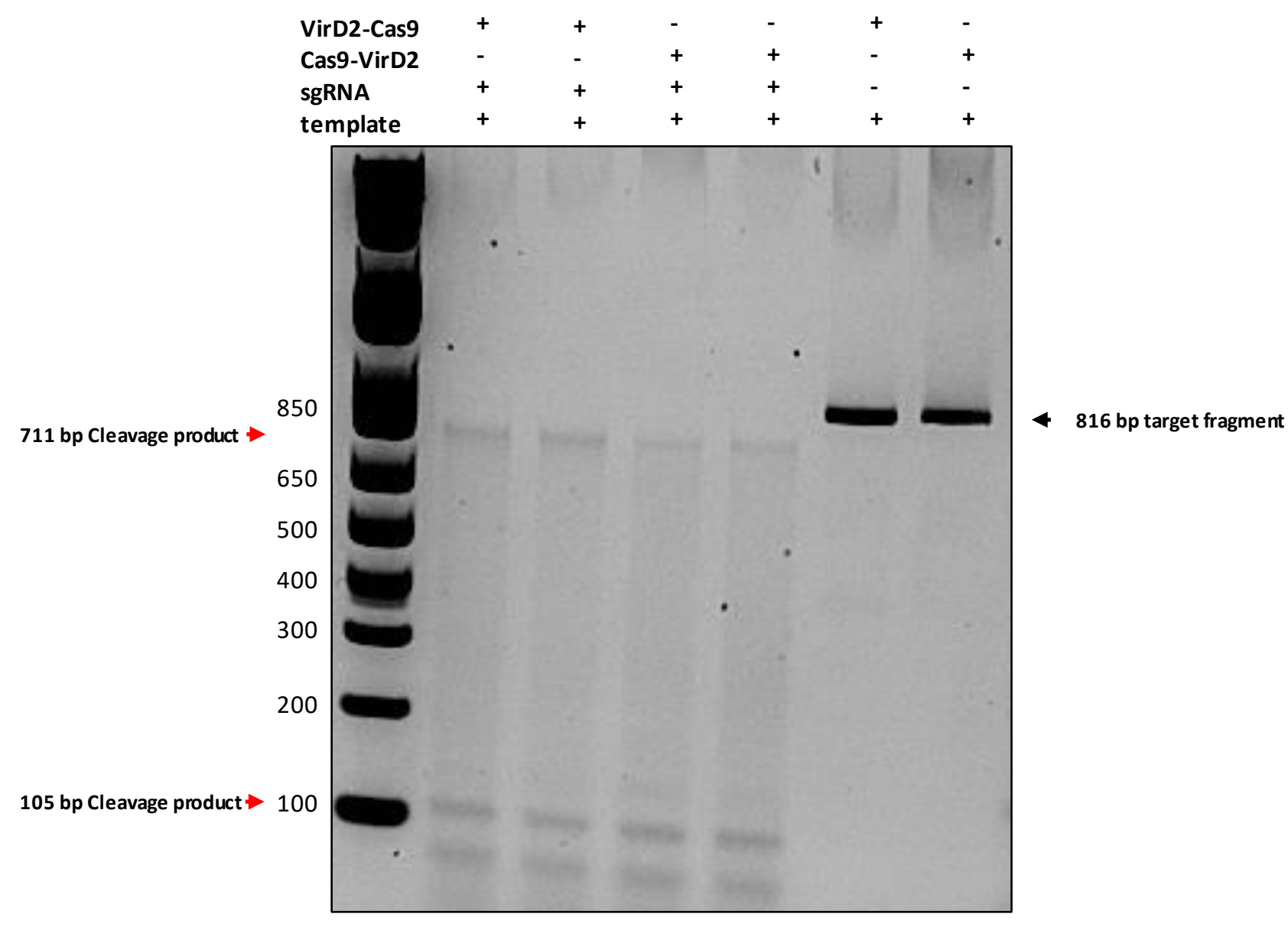

Supplementary Figure 4. Confirmation of VirD2-Cas9 and Cas9-VirD2 nuclease activity. N-gene target DNA was incubated with fusion proteins in the presence sgRNA and $\mathrm{Mg}^{2+}$. Samples without sgRNA were used as controls. Arrow heads, indicate the respective DNA fragments. 


\section{$\mathrm{N}$-gene target 1}

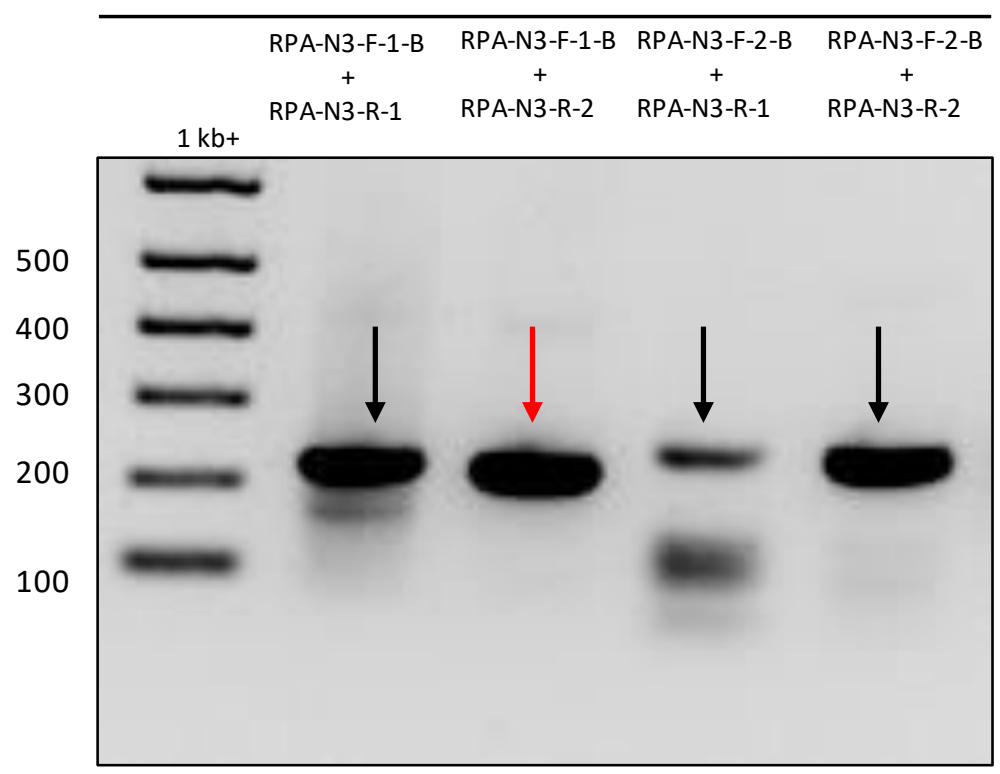

N-gene target 2

RPA-SC-F-1-B RPA-SC-F-1-B RPA-SC-F-2-B RPA-SC-F-2-B

$\begin{array}{cccc}+ & + & + & + \\ \text { RPA-SC-R-1 } & \text { RPA-SC-R-2 } & \text { RPA-SC-R-1 } & \text { RPA-SC-R-2 }\end{array}$

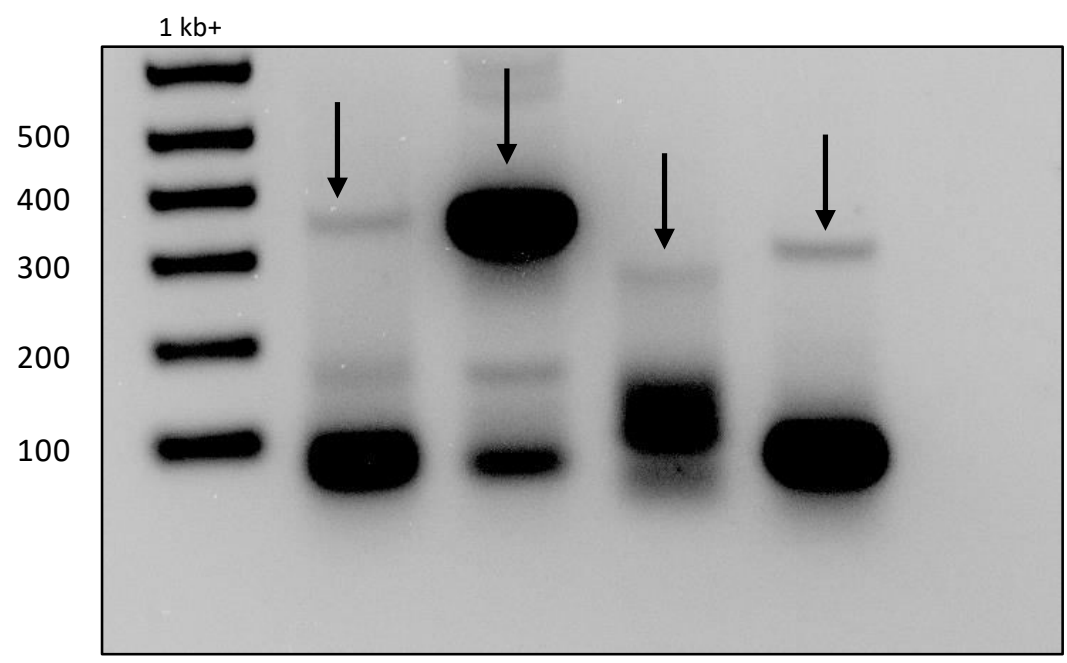

Supplementary Figure 5. Selection of the optimal RT-RPA primer set. N-gene target DNA was amplified by RT-RPA kit using manufacturer's instructions. Black arrow heads indicate the expected amplicons. Red arrow head, indicates the selected primer set. Primer set was selected based on the absence of non-specific amplicons and primer dimers. 


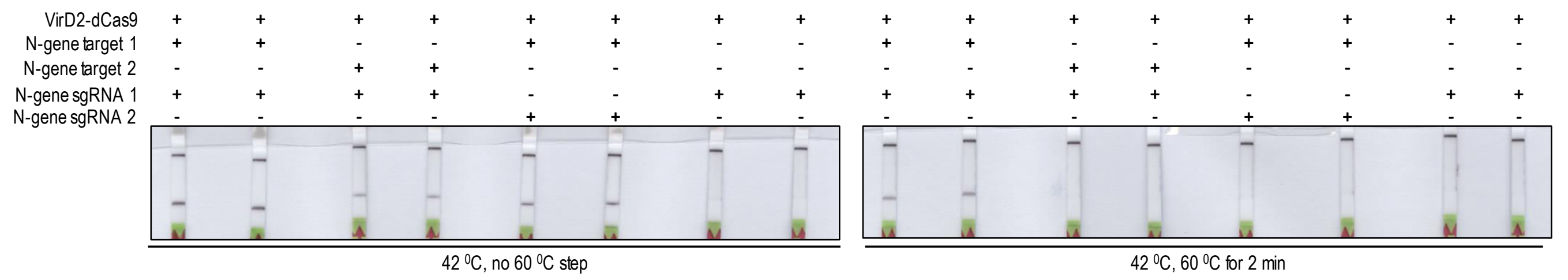

Supplementary Figure 7. Evaluation of the Vigilante at $42{ }^{\circ} \mathrm{C}$. All steps, the RT-RPA reaction, reporter complex assembly and detection reaction were carried out at $42^{\circ} \mathrm{C}$. N-gene specific target and specific sgRNA were used as positive and no template samples was used as negative control. 
N-gene sgRNA 2

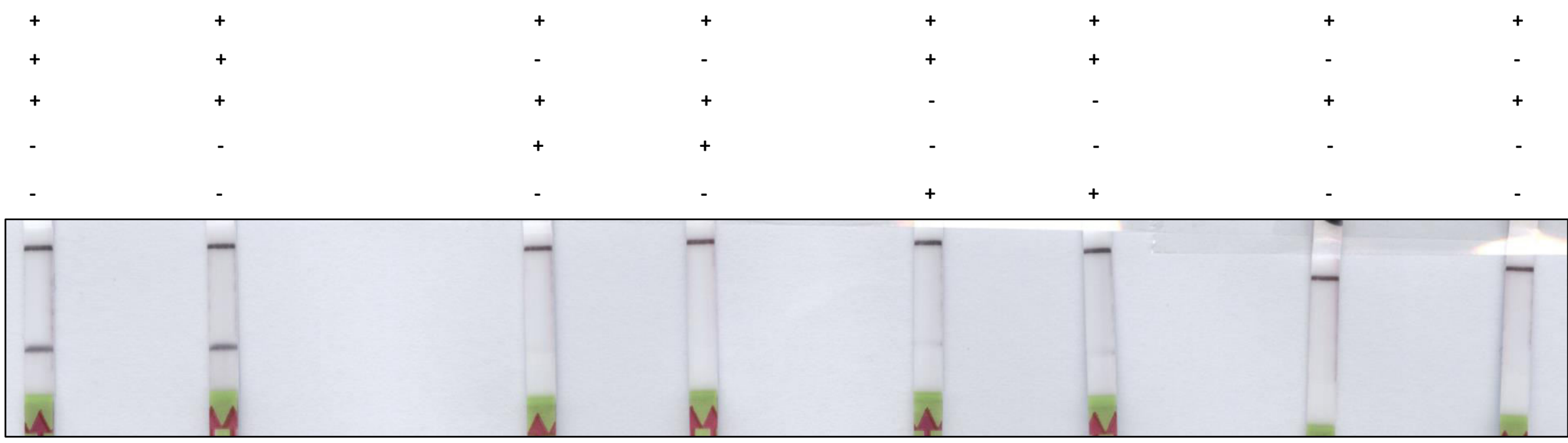

VirD2-dCas9

$\mathrm{N}$-gene target 1

$\mathrm{N}$-gene sgRNA 1

$\mathrm{N}$-gene target 2

$\mathrm{N}$-gene sgRNA 2

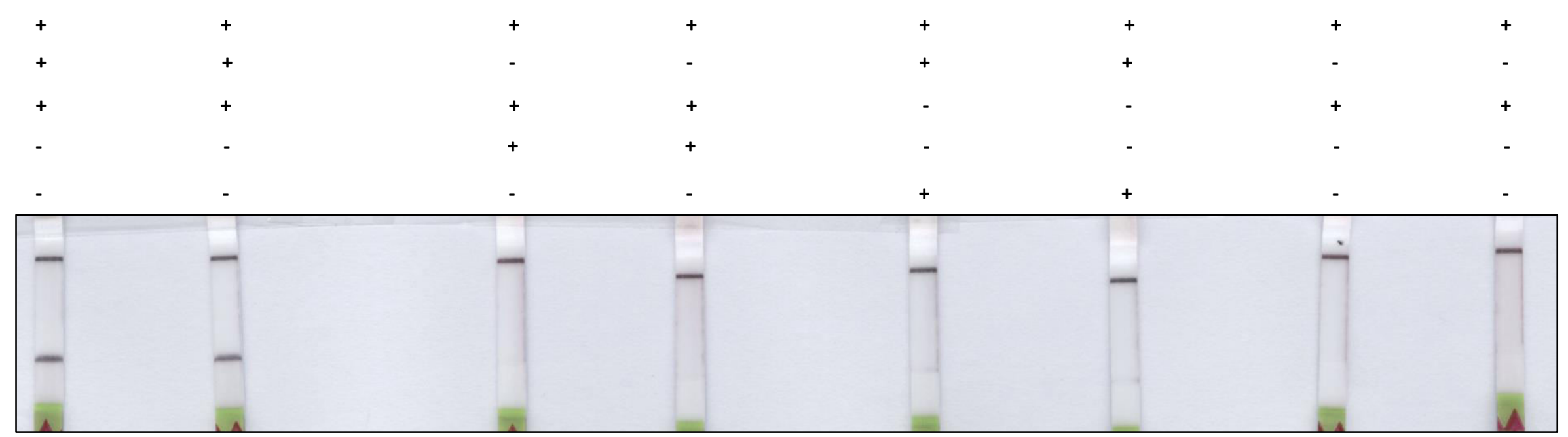

Supplementary Figure 8. Comparison of VirD2-Cas9 and VirD2-dCas9. SARS-CoV-2 RT-RPA amplified N-gene product was detected with the Vigilante platform. N-gene target 1, N-gene specific target; N-gene target 2, N-gene non-specific target; N-gene sgRNA 1, specific sgRNA; N-gene sgRNA 2, non-specific sgRNA 


\section{Replicates of the LoD experiment conducted in Figure 3A}

\section{Replicate 2}

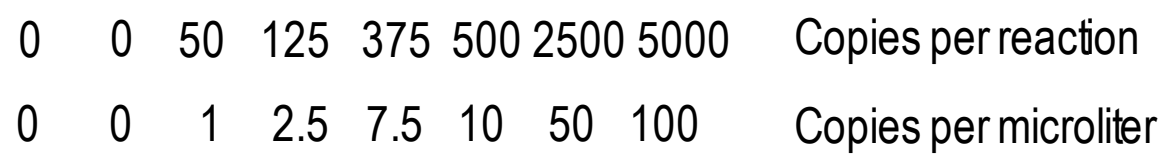

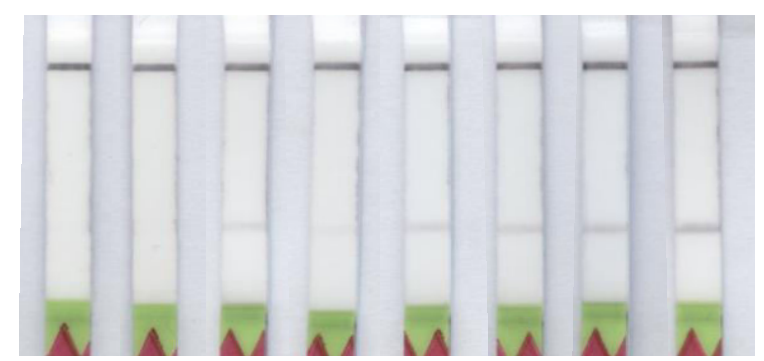

Replicate 3
$0 \quad 0 \quad 50125 \quad 37550025005000$
Copies per reaction
$\begin{array}{llllllll}0 & 0 & 1 & 2.5 & 7.5 & 10 & 50 & 100\end{array}$
Copies per microliter

Supplementary Figure 9. Replicates of LoD experiment conducted in Figure 3A 


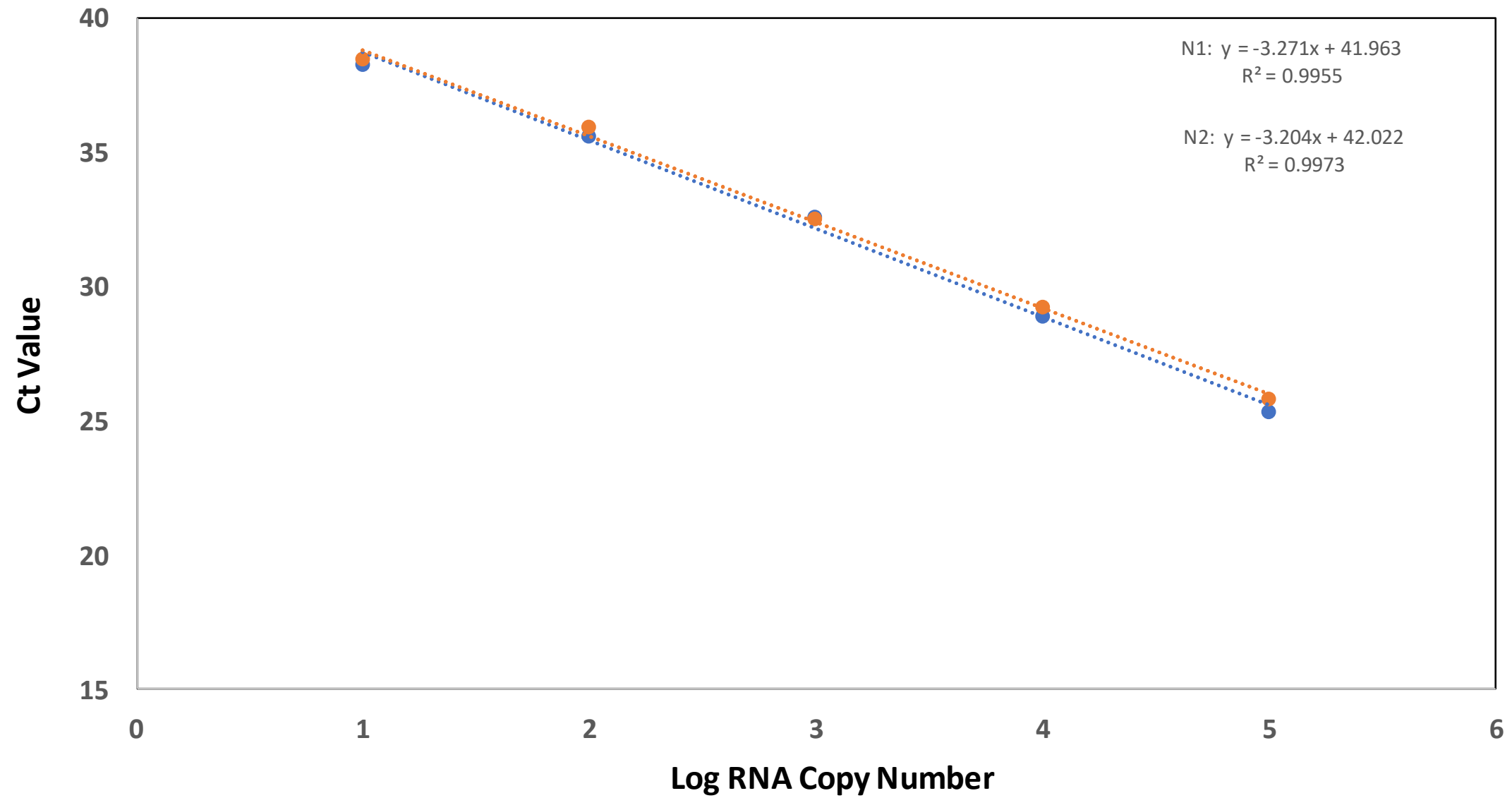

Supplementary Figure 10. Copy number determination by RT-qPCR for Ct value relevance in clinical samples. Synthetic SARS-CoV-2 RNA template was used to determine the LOD by RT-qPCR using One-step RT-qPCR kit (Invitrogen). Ct value was determined using two independent sets primer sets, N1 and N2. Blank sample (no template) was used as negative control. 


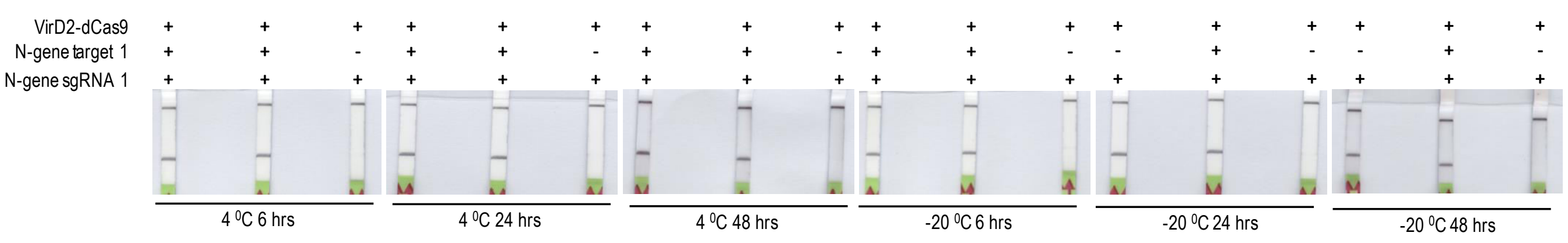

Supplementary Figure 11. Reporter complex stability. Protein:sgRNA:FAM-probe were mixed in 1:1:1 ratio in 250 , nM concentration and incubated at $37{ }^{\circ} \mathrm{C}$ for 60 minutes. The reporter complex was stored at different temperatures for different periods of time. N-gene specific target and specific sgRNA were used as positive and no template samples was used as negative control. 

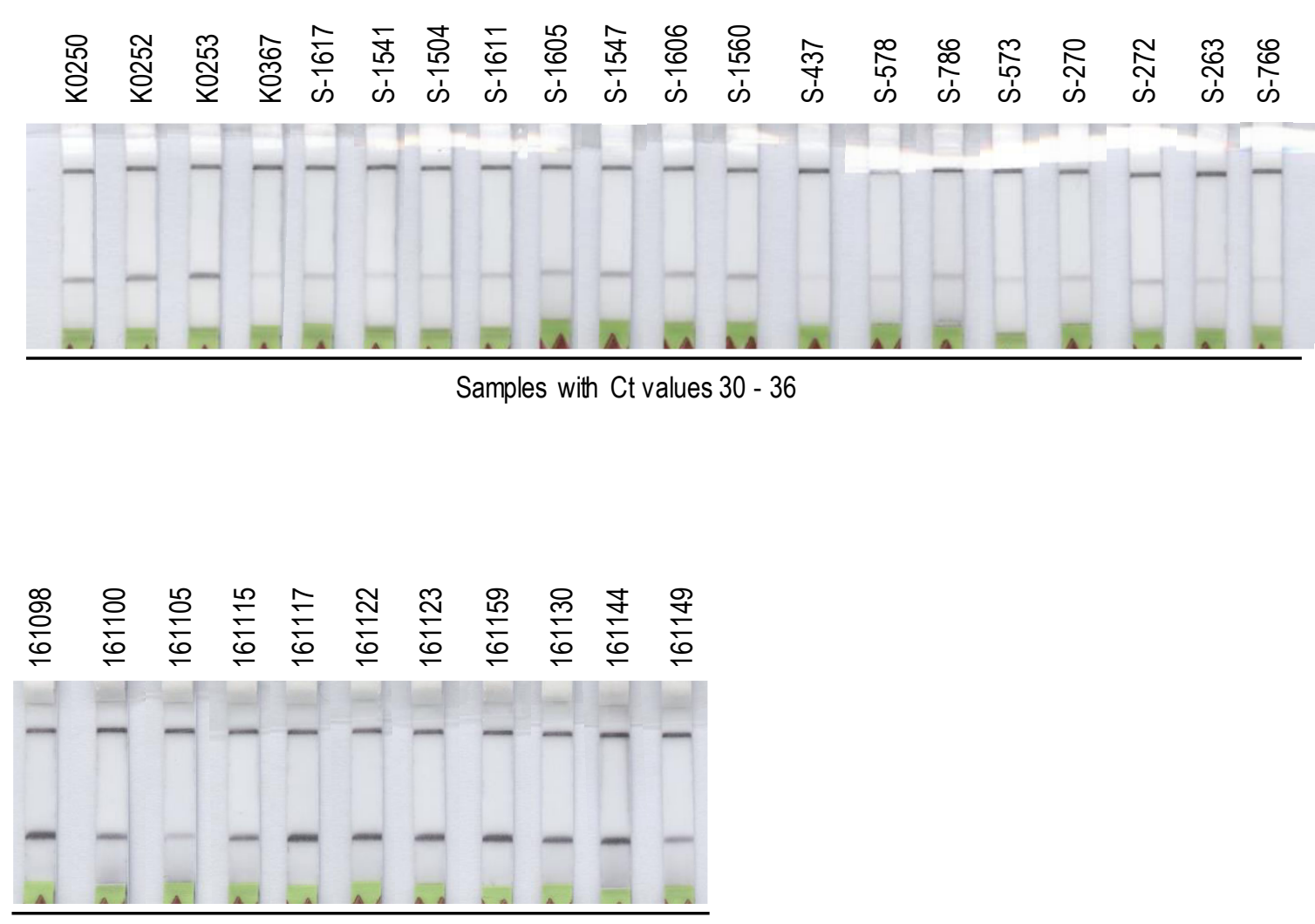

Samples with Ct values bellow 30

Supplementary Figure 12. Additional evaluated clinical samples and their original scan. 


\begin{tabular}{|l|l|}
\hline Sample designation & \multicolumn{1}{|c|}{ Ct value (RT-qPCR) } \\
\hline 155418 & 21 \\
\hline 155378 & 34 \\
\hline 155381 & 21 \\
\hline 155383 & 21 \\
\hline 155384 & 14 \\
\hline 155385 & 22 \\
\hline 155388 & 22 \\
\hline 155389 & 23 \\
\hline 155369 & 16 \\
\hline 155397 & 18 \\
\hline 155398 & 23 \\
\hline 155902 & 15 \\
\hline 155905 & 20 \\
\hline 155908 & 17 \\
\hline 155916 & 22 \\
\hline 155960 & 15 \\
\hline 155974 & 26 \\
\hline 156852 & 21 \\
\hline 161058 & 17 \\
\hline 161063 & 16.5 \\
\hline S1504 & 30.75 \\
\hline S1611 & 30.77 \\
\hline S1605 & 31.12 \\
\hline S1547 & 31.45 \\
\hline S1606 & 31.52 \\
\hline S1560 & 31.57 \\
\hline NID-1 & $>38$ \\
\hline NID-2 & 338 \\
\hline NID-3 & $>38$ \\
\hline NID-4 & 338 \\
\hline & \\
\hline
\end{tabular}

\begin{tabular}{|c|c|c|c|}
\hline Sample & Ct value & Sample & Ct value \\
\hline K0250 & 34.64 & 161098 & 29 \\
\hline K0252 & 36.06 & 161100 & 20 \\
\hline K0253 & 35.57 & 161105 & 23 \\
\hline K0367 & 35.16 & 161115 & 16 \\
\hline S-1617 & 30.00 & 161117 & 15 \\
\hline S-1541 & 30.55 & 161122 & 19 \\
\hline S-1504 & 30.75 & 161123 & 22 \\
\hline S-1611 & 30.77 & 161159 & 21 \\
\hline S-1605 & 31.12 & 161130 & 18 \\
\hline S-1547 & 31.45 & 161144 & 23 \\
\hline S-1606 & 31.52 & 161149 & 19 \\
\hline S-1560 & 31.57 & & \\
\hline S-437 & 32.47 & 33.25 & \\
\hline S-578 & & \\
\hline S-786 & 32.88 & & \\
\hline
\end{tabular}

Supplementary table 1. Designation and the Ct value of the clinical samples used in this study. RT-qPCR was performed on Trizol based isolated RNA. 


\begin{tabular}{|c|c|c|}
\hline Name & Sequence & Experimental description \\
\hline $\begin{array}{l}\text { virD2 reporter- } \\
\text { FAM-S }\end{array}$ & 5'-/gtttacccgccaata tatcctgtca TाTाT/56-FAM/-3' & Short, FAM-labeled probe for VirD2 \\
\hline $\begin{array}{l}\text { virD2 reporter- } \\
\text { FAM-L }\end{array}$ & 5'-/gtttacccgccaata tatcctgtca TाTाTाTाT/56-FAM/-3' & Long, FAM-labeled probe for VirD2 \\
\hline virD2 reporter-B-L & 5'-/gtttacccgccaatatatcctgtca TाTाTाTाT/biotin/-3' & $\begin{array}{l}\text { Long, biotin-labeled probe for VirD2 used for Westem- } \\
\text { blot analysis of VirD2 nuclease- and binding activities }\end{array}$ \\
\hline PCR-F1-N3 & 5'/biotin/CCGAAGAGCTACCAGACGAATTC/3' & Forward PCR primer used for proof-of-concept assay \\
\hline PCR-R2-N3 & 5'/TGTAGCACGATTGCAGCATTG/3' & Reverse PCR primer used for proof-of-concept assay \\
\hline $\begin{array}{l}\text { gBlock-N3-sgRNA- } \\
1\end{array}$ & $\begin{array}{l}\text { 5'/GTCTCAGGCATAATACGACTCACTATAGGccagaagctggacttccctaGTTTAGAGGCT } \\
\text { AGAAATAGCAAGTTAAAATAAGGCTAGTCCGTTATCAACTTGAAAAAGTGGCACCG } \\
\text { AGTCGGTGC/3' }\end{array}$ & $\begin{array}{l}\text { gBlock template used for IVT of sgRNA targeting both the } \\
\text { PCR and RT-RPA generated amplicons }\end{array}$ \\
\hline $\begin{array}{l}\text { RPA-STOPCovid-F- } \\
\text { 1-B }\end{array}$ & 5 '/biotin/actaagaaatctgctgctgaggcttctaag & Used for primer screening \\
\hline $\begin{array}{l}\text { RPA-STOPCovid-R- } \\
1\end{array}$ & $51 /$ tgcgtcaatatgcttattcagcaaaatgac & Used for primer screening \\
\hline $\begin{array}{l}\text { RPA-STOPCovid-F- } \\
\text { 2-B }\end{array}$ & 5'/biotin/gtactgccactaa agcatacaatgtaacac & Used for primer screening \\
\hline $\begin{array}{l}\text { RPA-STOPCovid-R- } \\
2\end{array}$ & 5'/gttttgta tgcgtcaatatgcttattcagc & Used for primer screening \\
\hline RPA-N3-F-1-B & 5'/biotin/agctaccagacgaattcgtggtggtgacgg & Selected RT-RPA fwd primer \\
\hline RPA-N3-R-1 & 5'/ttgtagcacgattgcagcattgttagcagg & Used for primer screening \\
\hline RPA-N3-F-2-B & 5'/biotin/aattggctactaccgaagagctaccagacg & Used for primer screening \\
\hline RPA-N3-R-2 & $5 ' /$ acgattgcagcattgttagcaggattgcgg & Selected RT-RPA revprimer \\
\hline pstv-cnt-RB-ssDNA & $\begin{array}{l}\text { cagatcaaTTCTCTTAGGTTTACCCGCCAATATATCCTGTCAAACACTGATAGTTTtca ca } \\
\text { gtagGGTG }\end{array}$ & Covalent binding confirmation of VirD2.dCas 9 fusions \\
\hline
\end{tabular}

Sequences of 3 different mutant proteins

>3_SpdCas9-dVirD2-Mutated

Ccaccctga attgactctcttccgggcgctatcatgcca taccgcgaaaggttttgcgccattcgatggtgtccgggatctcgacgctctcccttatgcgactcctgcattaggaagcagcccagtagtaggttgag gccgttgagcaccgccgccgcaaggaatggtgcatgcaaggaga tggcgcccaa cagtcccccggcca cggggcctgccaccatacccacgccgaaacaagcgctcatgagcccgaagtggcgagcccgatc ttccccatcggtgatgtcggcga tataggcgccagcaaccgcacctgtggcgccggtga tgccggccacgatgcgtccggcgtagaggatcgagatctcgatcccgcgaaattaatacgactcac tatagggga attgtgagcggataacaattcccctctagaaa taattttgttta actttaagaaggaga tataccatgggcagcagccatcatca tcatcatcacagcagcggcctggtgccgcgcggcagccatatggctagcat gactggtgga cagcaaatgggtcgcggatccgaattcga gctccgtcgaca agcttgcggccgcatggacaagaagtacagcatcggcctggCTatcggtaccaacagcgtgggctgggccgtgatcaccgac gagta caa ggtgcccagca agaagttcaaggtgctgggcaacaccgaccgccacagca tcaagaagaacctgatcggcgccctgctgttcgacagc ggcgagaccgccgaggccacccgcctga agcgcacc gcccgccgccgctaca cccgccgcaagaaccgcatctgctacctgcaggagatcttcagcaacgaga tggccaaggtggacgaca gcttcttccaccgcctggaggagagcttcctggtggaggaggacaaga agca cgagcgccaccccatcttcggcaacatcgtggacgaggtggcctaccacgagaagtaccccaccatctaccacctgcgcaagaagctggtggacagca ccgacaaggccgacctgcgcctga tctacct ggccctggcccacatgatcaa gttccgcggccacttcctgatcgagggcgacctgaa ccccgacaa cagcgacgtggacaagctgttcatccagctggtgcagacctacaacca gctgttcgagga gaacccca tca a cgccagcggcgtggacgcca aggccatcctgagcgcccgcctgagcaagagccgccgcctggagaacctgatcgcccagctgcccggcgagaagaagaa cggcctgttcggcaacctgatcgccctga gcctgggcctgacccccaacttca agagcaa cttcgacctggccga ggacgccaa gctgcagctga gcaaggacacctacgacgacgacctgga caacctgctggcccagatcggcga ccagtac gccgacct gttcctggccgccaagaacctgagcgacgccatcctgctgagcga catcctgcgcgtgaacaccgagatcaccaaggcccccctgagcgccagcatga tcaagcgcta cgacgagca ccaccaggacctgacc ctgctga aggccctggtgcgccagcagctgcccgaga agtacaaggagatcttcttcgaccagagcaagaacggctacgccggctacatcga cggcggcgccagccaggaggagttctacaagttcatcaagc ccatcctggaga agatgga cggcaccgaggagctgctggtgaagctgaa ccgcgaggacctgctgcgcaagcagcgcaccttcgacaacggcagcatcccccaccagatccacctgggcgagctgcacgccat cctgcgccgccaggaggacttcta ccccttcctgaa ggacaaccgcgagaaga tcgagaagatcctgaccttccgcatcccctactacgtgggccccctggcccgcggcaacagccgcttcgcct ggatgacccg ca aga gcgaggagaccatcaccccctggaa cttcgaggaggtggtggacaagggcgccagcgcccagagcttcatcgagcgcatgaccaacttcgacaagaacctgccca acgagaaggtgctgcccaagca cagcctgctgtacgagtacttcaccgtgtacaacgagctgaccaaggtgaagta cgtgaccgagggca tgcgcaagcccgccttcctgagcggcgagcagaagaaggcca tcgtggacctgctgt tcaagacca accgcaaggtgaccgtgaa gcagctgaa ggaggactacttcaaga agatcgagtgcttcgaca gcgtggagatcagcggcgtggaggaccgcttca acgccagcctgggcacctacca cgacctgctgaagat catca aggacaaggacttcctggacaacgaggagaacgaggacatcctggaggacatcgtgctgaccctgaccctgttcgaggaccgcgagatga tcgaggagcgcctgaagacctacgcccacctgttcgac gaca ggtga tgaagcagctgaagcgccgccgctacaccggctggggccgcctgagccgcaagctta tcaacggcatccgcgacaagcagagcggcaagaccatcctggacttcctgaagagcgacggcttc gccaa ccgcaacttcatgca gctgatcca cgacgacagcctgaccttcaaggaggacatccagaaggcccaggtgagcggccagggcgaca gcctgcacgagcacatcgccaacctggccggcagccccgcc atca aga agggcatcctgcagaccgtgaaggtggtgga cgagctggtgaaggtgatgggccgccacaa gcccgagaacatcgtgatcgagatggcccgcgagaacca gaccacccaga agggccagaaga a cagccgcgagcgcatgaagcgcatcgaggagggcatcaaggagctgggcagccagatcctga aggagca ccccgtggagaa cacccagctgca gaacgaga agctgta cctgtactacctgcagaa cggc cgcgaca tgtacgtggaccagga gctggaca tcaaccgcctgagcgacta cgacgtggacGCcatcgtgccccagagcttcctgaa ggacgacagca tcgacaa caaggtgctgacccgcagcgacaagaa ccgcggca agagcgacaacgtgcccagcgaggaggtggtgaagaagatgaagaactactggcgccagctgctgaacgcca agctgatcacccagcgcaagttcgaca acctgacca aggccgagcgcggcg gcctga gcgagctggacaaggccggcttcatcaagcgccagctggtggagacccgcca gatcaccaagcacgtggcccagatcctggacagccgcatgaacaccaagtacgacgagaacgacaagctgatcc gcgaggtgaaggtgatcaccctgaagagcaagctggtgagcgacttccgcaaggacttccagttctacaaggtgcgcgagatcaacaactaccaccacgcccacgacgcctacctgaacgccgtggtgggcac 
cgccctga tcaagaagtacccaagctggagagcgagttcgtgtacggcga ctacaaggtgtacga cgtgcgcaagatgatcgccaagagcgagca ggagatcggcaaggccaccgccaagtact tcttctac agca a catcatgaa cttcttcaa gaccgagatcaccctggcca acggcgagatccgcaagcgccccctgatcgaga ccaacggcgagaccggcgagatcgtgtgggacaagggccgcgacttcgccaccgtgc gca aggtgctga gcatgccccaggtgaaca tcgtgaaga agaccgaggtgcagaccggcggcttcagcaaggagagcatcctgcccaagcgcaacagcga caagctga tcgcccgcaagaaggac tgggac cccaa gaagtacggcggcttcgacagccccaccgtggcctacagcgtgctggtggtggccaaggtgga gaagggcaa gagcaaga agctgaagagcgtgaa ggagctgctgggcatcaccatcatggagcgc agca gcttcgagaagaa ccccatcgacttcctggaggcca agggctaca aggaggtga agaaggacctgatcatcaagctgcccaagtaca gcctgttcgagctggagaa cggccgcaagcgcat gctggcc agcgccggcgagctgcagaagggcaacgagctggccctgcccagcaa gtacgtga acttcctgta cctggccagcca ctacgaga agctgaagggcagccccgaggacaacgagcagaagcagctgttcgtg gagca gcacaagcactacctggacgaga tcatcgagcagatcagcgagttcagcaagcgcgtgatcctggccgacgccaacctggaca aggtgctga gcgcctacaa caagcaccgcga caagcccatccgc gagca ggccgagaacatcatccacctgttcaccctgacca acctgggcgcccccgccgccttcaagtacttcgacaccaccatcga ccgcaagcgctacaccagca ccaaggaggtgctggacgccaccctgat cca ccagagcatcaccggtctgtacgagacccgcatcgacctga gccagctgggcggcgacggcggctccgga cctccaaa gaaaaagagaaa agtatacccctacgacgtgcccgactacgcct gtggagg cggtgga tctggcggtggcggaagtgggggaggtgga agtggtaccatgcccgatcgagctcaagttatcattcgcattgtgccgggaggtggca ccaagacccttcaacaaattatcaatcagttggagGccc tatcccggaagggcaggctggagctgcagcgttcagcccgacatctcgatattcccctgccaccgga tcaaatccacgaacttgcccgaagctgggttcaagagactgga acttatgacgaaagt cagccaga $c$ gagga a agcaacaggagttgacca cccatattattgtaagcttccccgccggtacaagcca ggtagcggctta tgcggcgagccggga gtgggcagccgagatgtttgggtcaggcgcaggggggggccgat aca actatcttacggccttccacatcgatcgcgaccacccacatctgca tgtcgtcgtca atcggcgcgaacttttaggacacggctggctgaagatatctcggcgcca tccccaactgaattac gacgccctgcg cata aa gatggccgaga tttcacttcgtcatggca ttgccctcga tgcgagccgacgagcagaacgtggcatcaccga gcggccgatca cttatgccca atatcggcgccttgagcgggagcaggctcgcca aa tccgtttcgaagacgcggatttggaacagtcgtcgccgcaaggagatca tccagaattcagccaa cctttcgatacatccccatttgaagcatccgcgggcgga ccggaggaca tgcctcggcccaacaatcggc a ga a tgagtcgcaagttcatctcca ggagccagctggtgtcagcaa cgaagccggtgtccttgtgcgggttgcattggagacggagcgccttgctcaacca ttcgtttccgaaacca ttctcgcggacga catagg gagcggctcttcgcgtgttgccgagggccgtgtggagagcgcaaaccgcactcccgatattcctcgcgcagcaactgaagctgccacgca cacgacacacgaccggcagcggcgtgcaaagcgtc ctcatgatg acga cggagggccgagtggagcaaaacgtgtgacattggaaggcatcgcggttgggccccaggcgaacgccggcgaacaggatggcagtagtggccccttagta cggcaagctggaacgtctcggccatctc ca ccga cggccacgacgcgggccagcaccgcaaccgattcattgtctgctacagcccacctccagcaa cggagaggtgtcctttcaaagcgtccgcgtgaagatga tgatggagaaccgagtgaacgcaa acg cgaga ga gatgagcgcagca aggacgggcgtgggggaaataggagataataa gcggccgcactcga gcaccaccaccaccaccactgagatccggctgctaacaaagcccgaaaggaagctga gttggctg ctgcca ccgctga gcaataactagcata accccttggggcctctaaacgggtcttgaggggtttttgctgaaaggaggaactatatccggattggcgaatgggacgcgccctgtagcggcgcat taagcgcggc gggtgtggtggtta cgcgcagcgtgaccgctacacttgccagcgccctagcgccegctcctttcgctttcttcccttcctttctcgccacgttcgccggctttccccgtcaagctctaa atcgggggctccctttagggt tccgatttagtgcttta cggcacctcgaccccaaaaa act tgattagggtga tggttcacgtagtgggccatcgccctgatagacggttttcgccctttgacgttggagtccacgttctttaat agtggactcttgttc ca a a ctggaacaaca ctcaaccctatctcggtcta ttcttttgattta taaggga ttttgccgatttcggcctattggttaa aaaatgagctgatttaaca aaaatttaacgcgaattttaacaaa atattaacgctta caatttaggtggcacttttcggggaa atgtgcgcggaacccctatttgtttatttttcta aatacattcaaatatgtatccgctcatgaattaa ttcttaga aaaactcatcgagcatcaaatgaaactgcaatttatt cata tcaggattatcaataccatatttttgaaaaagccgtttctgtaatga aggagaaaactcaccgaggcagttccataggatggcaaga tcctggtatcggtctgcgattccgactcgtcca a catca atacaa cctattaatttcccctcgtcaaaa ataaggttatcaagtgagaaatcaccatgagtgacga ctgaatccggtgagaatggcaaaagtttatgcatttctttccagacttgttcaaca ggccagccattacgctcgtc atca aa atcactcgcatcaacca aaccgttattcattcgtgattgcgcctgagcgagacgaaatacgcgatcgctgttaaaaggacaattacaaacaggaatcgaatgca accggcgcaggaacactgccagc gca tca aca atattttcacctgaatcagga tattcttctaatacctggaatgctgttttcccgggga tcgcagtggtgagtaa ccatgcatcatcaggagtacggata aaatgcttgatggtcggaagaggcataa attccgtca gccagttta gtctgaccatctcatctgtaacatcattggcaacgctacctttgccatgtttca gaaaca actctggcgcatcgggcttccca tacaatcgatagattgtcgcacct gattgcccga catt atcgcgagcccatttatacccatataaatcagcatccatgttggaatttaatcgcggcctagagcaagacgtttcccgttgaatatggctcataacaccccttgtattactgtttatgtaagcagacagttttattgtt catga ccaa aatcccttaacgtga gttttcgttccactgagcgtcagaccccgta gaaaagatcaaa ggatcttcttgagatcctttttttctgcgcgtaa tctgctgcttgcaaacaa aaaaac caccgcta ccag cggtggtttgtttgccggatcaagagccacca actcttttccgaaggtaactggcttcagcaga gcgcagata ccaaata ctgtccttctagtgtagccgtagttaggccaccacttca agaactctgtagcaccg ccta catacctcgctctgcta atcctgttaccagtggctgctgccagtggcga taagtcgtgtcttaccgggttggactca agacgatagttaccgga taaggcgca gcggtcgggctga acggggggttcgtgca ca ca gcccagcttggagcgaa cgacctaca ccgaactgagataccta cagcgtgagctatgaga aagcgccacgcttcccgaaggga gaaaggcggacaggtatccggtaagcggcagggtcggaacagga gagcgca cgagggagcttccagggggaaacgcctggtatctttatagtcctgtcgggtttcgccacctctgacttgagcgtcgatttttgtgatgctcgtcaggggggcggagcctatggaaa aacgcca gcaacg cggccttttta cggttcctggccttttgctggccttttgctcacatgttctttcctgcgttatcccctgattctgtggata accgtattaccgcctttga gtgagctga taccgctcgccgca gccgaacga ccgagcgca gcgagtcagtga gcgaggaa gcggaaga gcgcctgatgcggtattttctccttacgcatctgtgcggtatttcacaccgcaatggtgca ctctcagtacaatctgctctgatgccgcatagtt aagccagtatacac tccgctatcgctacgtgactgggtcatggctgcgccccgacacccgcca acacccgctgacgcgccctgacgggcttgtctgctcccggca tccgcttacagacaagctgtgaccgtctccgggagctgcatgtgtc agaggttttcaccgtcatcaccgaaacgcgcgaggcagctgcggtaaagctcatcagcgtggtcgtgaagcgattca cagatgtctgcctgttcatccgcgtccagctcgttgagtttctccagaagcgttaatgtc tggcttctga taaagcgggccatgttaagggcggttttttcctgtttggtcactgatgcctccgtgta agggggatttctgttca tgggggtaatgataccga tgaaacgagagagga tgctcacga tacgggttact ga tga tga acatgcccggttactggaacgttgtga gggtaaacaactggcggta tggatgcggcgggaccagaga aaaatcactcagggtcaatgccagcgcttcgttaataca gatgtaggtgttccacaggg ta gcca gcagcatcctgcgatgcagatccggaacata atggtgcagggcgctgacttccgcgtttccaga ctttacga aacacggaaaccgaa gaccattca tgttgttgctcaggtcgcagacgttttgcagca gcagtcgcttcacgttcgctcgcgtatcggtgattcattctgctaaccagtaaggcaaccccgccagcctagc cgggtcctcaacgacaggagcacgatcatgcgcacccgtggggccgccatgccggcgataat ggcctgcttctcgccgaaacgtttggtggcgggaccagtgacgaa ggcttgagcgagggcgtgcaagattccgaa taccgcaagcgacaggccga tcatcgtcgcgctccagcgaa agcggtcctcgccgaaa atga cccaga gcgctgccggcacctgtcctacga gttgcatgataaagaagacagtcataagtgcggcgacgatagtcatgccccgcgcccaccggaa ggagctgactgggttgaaggctctcaagggcatcg gtcga ga tcccggtgcctaatgagtgagctaactta cattaa ttgcgttgcgctcactgcccgctttccagtcgggaaacctgtcgtgccagctgcattaatga atcggccaacgcgcggggagaggcggtttgcg tattgggcgcca gggtggtttttcttttca ccagtgaga cgggcaaca gctgattgcccttcaccgcctggccctgagagagttgcagcaagcggtccacgctggtttgccccagca ggcgaaaa tcctgtttgatg gtggtta a cggcgggatataacatgagctgtcttcggtatcgtcgtatcccactaccgagatatccgcaccaa cgcgcagcccggactcggtaatggcgcgcattgcgcccagcgccatctga tc gttggcaacca gcatcgcagtgggaacgatgccctcattcagcatttgcatggtttgttgaaaaccggacatggcactccagtcgccttcccgttccgcta tcggctgaatttgattgcgagtgaga tatttatgccagccagccaga cgcaga cgcgccga gacagaa cttaatgggcccgctaaca gcgcgatttgctggtgacccaatgcgacca gatgctccacgcccagtcgcgtaccgtcttcatggga gaaaataatactgttgat gggtgtctgg tcaga gacatcaaga aataa cgccggaacattagtgca ggcagcttccacagcaatggcatcctggtcatccagcggatagttaa tgatcagcccactgacgcgttgcgcgagaagattgtgca ccgccgcttta caggcttcgacgccgcttcgttctaccatcgacaccaccacgctggcacccagttgatcggcgcgagatttaatcgccgcgacaatttgcgacggcgcgtgcagggccagactggaggtggcaacgccaatcag ca a cgactgtttgcccgccagttgttgtgccacgcggttgggaa tgtaattcagctccgccatcgccgcttccactttttcccgcgttttcgcagaaacgtggctggcctggttcaccacgcgggaaa cggtctgata aga ga caccggcatactctgcgacatcgtataacgttactggtttcacattca

\section{>5_VirD2-SpdCas9-Mutated}

ttta acgcgaattttaacaa aatattaacgcttacaatttaggtggcacttttcggggaa atgtgcgcggaacccctatttgttta tttttctaaata cattcaaatatgtatccgctcatgaattaattcttagaaaaa ctcatcgagca tcaaatgaaactgcaatttattcatatcaggattatcaataccata ttttga aaaagccgtttctgta atgaa ggagaaaactcaccgaggcagttccataggatggcaagat cctggtatcggt ctgcgattccgactcgtccaa catcaatacaaccta ttaatttcccctcgtcaaaaataaggtta tcaagtgagaaatcaccatgagtgacgactgaatccggtgagaatggca aaagtttatgcatttctttccag acttgttcaacaggccagccattacgctcgtcatcaa aatcactcgcatcaa ccaaaccgttattcattcgtgattgcgcctgagcgagacgaaa tacgcgatcgctgttaaaaggacaattacaaacaggaatc ga a tgcaa ccggcgcaggaacactgccagcgcatcaacaatattttcacctga atcaggatattcttctaatacctggaatgctgttttcccggggatcgcagtggtgagtaaccatgcatca tcaggagtacgga ta a a atgcttgatggtcggaagaggcataaattccgtca gccagtttagtctgaccatctcatctgtaacatcattggcaacgctacctttgccatgtttca gaaaca actctggcgcatcgggcttccca tacaatc ga tagattgtcgcacctgattgcccgacattatcgcgagcccattta tacccatataaa tcagcatccatgttggaatttaatcgcggcctagagcaagacgtttcccgttga atatggctcataa caccccttgtat tactgtttatgtaagcagacagttttattgttcatga ccaaaatcccttaacgtgagttttcgttccactgagcgtcagaccccgtagaaaagatca aaggatcttcttgagatccttttttctgcgcgtaatctgctg 
cttgca aa caaaaa aaccaccgctaccagcggtggtttgtttgccggatcaa gagccaccaactctttttccgaaggta actggcttcagcagagcgcagataccaaatactgtccttctagtgtagccgtagtta ggcca ccacttca agaactctgtagcaccgcctacata cctcgctctgctaatcctgttaccagtggctgctgccagtggcgataagtcgtgtcttaccgggttggactcaagacgatagttaccggataaggcgca gcggtcgggctga acggggggttcgtgcacacagccca gcttggagcgaacgaccta caccgaactgagatacctacagcgtgagctatgagaaagcgccacgcttcccgaagggagaaa ggcggacaggta tccggtaagcggcagggtcggaacaggagagcgcacgagggagcttccagggggaaacgcctggtatctttatagtcctgtcgggtttcgcca cctctgacttgagcgtcgatttttgtga tgctcgtcagggggg cgga gcctatggaaaaa cgccagcaacgcggcctttttacggttcctggccttttgctggccttttgctcacatgttctttcctgcgttatcccctgattctgtggataaccgtattaccgcctttgagtgagctgatac cgctcgccgcagccgaacgaccgagcgcagcgagtcagtgagcgagga agcggaaga gcgcctgatgcggtattttctccttacgca tctgtgcggtatttcacaccgcaatggtgcactctca gtacaatctgc tctgatgccgcatagttaagccagtatacactccgctatcgctacgtgactgggtca tggctgcgccccgacacccgccaacacccgctgacgcgccctgacgggcttgtctgctcccggcatccgcttacaga ca agctgtga ccgtctccgggagctgcatgtgtcagaggttttcaccgtcatca ccgaaacgcgcgaggcagctgcggtaaagctcatcagcgtggtcgtgaagcgattcacagatgtctgcctgttcatccgcgtcc agctcgttgagtttctccagaagcgttaatgtctggcttctgataaagcgggccatgttaagggcggttttttcctgtttggtcactgatgcctccgtgtaa gggggatttctgttcatgggggt aatga taccgatga a a cga gagaggatgctca cgatacgggttactgatgatgaa catgcccggtta ctggaacgttgtgagggtaaacaactggcggtatggatgcggcgggaccagagaa aaatcactcagggtcaatgccagcg cttcgttaa tacagatgtaggtgttccacagggtagccagcagcatcctgcgatgcagatccggaacataa tggtgcagggcgctgacttccgcgtttccagactttacgaa acacggaaaccgaaga ccattca tgttgttgctcaggtcgcaga cgttttgcagcagcagtcgcttcacgttcgctcgcgtatcggtgattcattctgctaaccagtaaggcaaccccgccagcctagccgggtcctcaacgacaggagcacgatcatgc gca cccgtggggccgccatgccggcgataatggcctgcttctcgccgaaacgtttggtggcgggaccagtga cgaaggcttgagcgagggcgtgcaagattccgaataccgcaagcgacaggccgatca tcgtc gcgctcca gcgaaagcggtcctcgccgaaaatgacccagagcgctgccggcacctgtcctacgagttgcatgataaagaa gacagtca taagtgcggcgacgata gtcatgccccgcgccca ccggaaggagc tga ctgggttgaaggctctcaagggcatcggtcgagatcccggtgcctaatgagtgagctaactta cattaa ttgcgttgcgctcactgcccgctttccagtcgggaaacctgtcgtgccagctgcattaatga atc ggcca a cgcgcggggagaggcggtttgcgtattgggcgccagggtggtttttctttcaccagtgagacgggcaacagctgattgcccttcaccgcctggccctgagaga gttgcagcaagcggtccacgctggtt tgcccca gcaggcgaaaatcctgtttgatggtggttaacggcgggatataacatgagctgtcttcggtatcgtcgtatcccactaccgagatat ccgcaccaa cgcgcagcccggactcggtaatggcgcgcattg cgcccagcgccatctgatcgttggca accagca tcgcagtgggaacgatgccctcattcagca tttgcatggtttgttga aaaccggacatggcactccagtcgccttcccgttccgctatcggctgaatttgattgc ga gtga ga tattta tgccagccagcca gacgcagacgcgccgagacagaacttaatgggcccgcta acagcgcgatttgctggtgacccaatgcgaccagatgctccacgcccagtcgcgtaccgtcttcatgg ga ga a aa taatactgttgatgggtgtctggtcagagaca tcaagaaataacgccggaacattagtgcaggcagcttccacagcaatggcatcctggtca tccagcggata gttaatgatcagccca ctgacgcg ttgcgcga gaa gattgtgcaccgccgcttta caggcttcga cgccgcttcgttctaccatcgacaccaccacgctggcacccagttgatcggcgcga gatttaa tcgccgcgacaatttgcga cggcgcgtgcagg gccaga ctgga ggtggcaacgccaatcagca acgactgtttgcccgccagttgttgtgccacgcggttgggaa tgtaattcagctccgccatcgccgcttccactttttcccgcgttttcgcagaaacgtggctggc ctggttca ccacgcgggaaacggtctgata agagaca ccggcatact tgcgacatcgtataacgttactggtttcacattcaccaccctgaattgactctcttccgggcgctatcatgccataccgcgaa aggttt tgcgccattcgatggtgtccgggatctcgacgctctcccttatgcgactcctgcattaggaagcagcccagtagtaggttgaggccgttgagcaccgccgccgcaaggaatggtgcatgcaagga gatggcgccc a a ca gtcccccggccacggggcctgcca ccatacccacgccgaaaca agcgctcatgagcccgaagtggcgagcccgatcttccccatcggtgatgtcggcgatataggcgccagcaaccgcacctgtggcgc cggtga tgccggccacgatgcgtccggcgtagaggatcgagatctcgatcccgcgaa attaatacgactcactataggggaattgtgagcggata acaattcccctctaga aataa ttttgtttaactttaagaag ga gata taccatgggcagcagccatcatcatcatcatcacagcagcggcctggtgccgcgcggcagccata tggctagcatgactggtgga cagcaaatgggtcgcgga tccgaattcgagctccgtcgacaag cttgcggccgcatgcccgatcgagctcaagtta tcattcgcattgtgccgggaggtggcaccaagacccttcaacaaattatcaa tcagttggagtatcta tcccggaagggcaggctggagctg cagcgttcagc ccga catctcgatattcccctgccaccggatcaaatccacgaacttgcccgaagctgggttcaagaga ctggaacttatgacgaaagtca gccagacgagga aaggca acaggagttgaccaccca tat tattg ta a gcttccccgccggtacaagccaggtagcggcttatgcggcgagccgggagtgggcagccgagatgtttgggtca ggcgcaggggggggccgatacaa ctatcttacggccttccaca tcgatcgcga ccac cca catctgcatgtcgtcgtcaatcggcgcga actttta ggacacggctggctgaagatatctcggcgccatccccaactgaatta cgacgccctgcgcataaagatggccgagatttcacttcg tcatggcattgc cctcga tgcgagccgacgagcagaacgtggcatcaccgagcggccgatcacttatgcccaatatcggcgccttgagcgggagcaggctcgccaaatccgtttcgaa gacgcggatttggaaca gtcgtcgccgc a agga ga tcatccaga attcagcca acctttcgatacatcccatttgaagcatccgcgggcggaccggaggacatgcctcggcccaacaatcggcagaatga gtcgcaagttcatctccaggagccagctggt gtca gca acga agccggtgtccttgtgcgggttgcattggaga cggagcgccttgctcaaccattcgtttccga aaccattctcgcggacgacataggga gcggctcttcgcgtgttgccgagggccgtgtggaga gcgca a accgcactcccgata ttcctcgcgcagcaactgaagctgccacgcacacgacacacgaccggcagcggcgtgcaaagcgtcctcatgatgacgacggagggccgagtggagcaaaacgtgtgacat tgga agca tcgcggttgggcccca ggcgaacgccggcga acagga tggcagtagtggccccttagtacggcaagctggaacgtctcggccatctcca ccgacggccacgacgcgggccagcaccgca accg attcattgtctgctaca gcccacctccagcaacggaga ggtgtcctttcaaagcgtccgcgtgaagatgatgatggagaaccgagtgaacgca aacgcgagagagatgagcgcagcaaggacgggcgtgggg ga a a taggagaaga tctggaggcggtggatctggcggtggcggaagtgggggaggtggaagtatgga caagaa gtacagca tcggcctggCTatcggtaccaacagcgtgggctgggccgtgatcaccgac ga gta caa ggtgcccagca agaagttcaaggtgctgggcaacaccgaccgccacagca tcaagaagaacctgatcggcgccctgctgttcgacagcggcgagaccgccgaggccacccgcctga agcgcacc gcccgccgccgctaca cccgccgcaagaaccgcatctgctacctgcaggagatcttcagcaacgaga tggccaaggtggacgaca gcttcttccaccgcctggaggagagcttcctggtggaggaggacaaga agca cgagcgccacccatcttcggcaacatcgtggacgaggtggcctaccacgagaagtaccccaccatctaccacctgcgcaagaagctggtggacagca ccgacaaggccgacctgcgcctga tctacct ggcctggcccacatgatcaa gttccgcggccacttcctgatcgagggcgacctgaa ccccgacaa cagcgacgtggacaagctgttcatccagctggtgcagacctacaacca gctgttcgagga gaacccca tca a cgccagcggcgtggacgcca aggccatcctgagcgcccgcctgagcaagagccgccgcctggagaacctgatcgcccagctgcccggcgagaagaagaa cggcctgttcggcaacctgatcgccctga gcctgggcctgaccccaacttca agagcaa cttcgacctggccga ggacgccaa gctgcagctga gcaaggacacctacgacgacgacctgga caacctgctggcccagatcggcga ccagtacgccgacct gttcctggccgccaagaacctgagcgacgccatcctgctgagcga catcctgcgcgtgaacaccgagatcacca aggccccctgagcgccagcatga tcaagcgcta $c g a c g a g c a c c a c c a g g a c c t g a c c$ ctgctga aggccctggtgcgccagca gctgcccgaga agtacaaggagatcttcttcgaccagagcaagaacggctacgccggctacatcga cggcggcgccagcca ggaggagttctacaagttcatcaagc ccatcctggaga agatgga cggcaccgaggagctgctggtgaagctgaa ccgcgaggacctgctgcgcaagcagcgcaccttcgacaacggcagcatcccccaccagatccacctgggcgagctgcacgccat cctgcgccgccagga ggacttcta ccccttcctgaa ggacaaccgcgagaaga tcgagaagatcctgaccttccgcatcccctactacgtgggcccctggcccgcggcaacagccgcttcgcctggatgacccg ca a ga gcga ggagaccatcaccccctggaa cttcgaggaggtggtggacaagggcgccagcgcccagagcttca tcgagcgcatgaccaacttcgacaagaacctgccca acgagaa ggtgctgc ccaagca cagcctgctgtacgagtacttcaccgtgtacaacgagctgaccaaggtgaagta cgtgaccgagggca tgcgcaagcccgcttcctgagcggcgagcagaagaaggcca tcgtggacctgctgttcaagacca a ccgca aggtgaccgtgaa gcagctgaa ggaggactacttcaaga agatcgagtgcttcgaca gcgtggagatcagcggcgtggaggaccgcttca acgccagcctgggcacctacca cgacctgctgaagat catca aggacaaggacttcctggacaacgaggagaacgaggacatcctggaggacatcgtgctgaccctgacctgttcgaggaccgcgagatga tcgaggagcgcctgaagacctacgcccacctgttcgac ga ca aggtga tgaagcagctgaagcgccgccgctacaccggctggggccgcctgagccgcaagctta tcaacggcatccgcgacaagcagagcggcaagaccatcctggacttcctgaagagcgacggcttc gccaa ccgcaacttcatgca gctgatcca cgacgacagcctgaccttcaaggaggacatccagaaggcccaggtgagcggccagggcgaca gcctgcacgagcacatcgccaacctggccggcagc cccgcc atca aga agggcatcctgcagaccgtgaaggtggtgga cgagctggtgaaggtgatgggccgccacaa gcccgagaacatcgtgatcgagatggcccgcgagaacca gaccacccaga agggccagaaga a ca gccgcgagcgcatgaagcgcatcgaggagggcatcaaggagctgggcagccagatcctga aggagca ccccgtggagaa cacccagctgca gaacgaga agctgta cctgtactacctgcagaa cggc cgcga ca tgtacgtggaccagga gctggaca tcaaccgcctgagcgacta cgacgtggacGCcat cgtgccccagagcttcctgaa ggacgacagca tcgacaa caaggtgctgacccgcagcgacaagaa ccgcggca agagcgacaacgtgcccagcgaggaggtggtgaagaagatgaagaactactggcgccagctgctgaacgcca agctgatcacccagcgcaagttcgaca acctgacca aggccgagcgcggcg gcctgagcgagctggacaaggccggcttcatcaagcgccagctggtggagacccgccagatcaccaagcacgtggccca gatcctggacagccgcatgaacaccaagtacgacgagaacgacaagctgatcc gcgaggtga aggtgatcacctgaagagcaagctggtgagcgacttccgcaaggacttccagttctacaaggtgcgcga gatcaacaactaccaccacgcccacgacgcctacctgaacgccgtggtgggcac cgccctgatcaagaagtacccaagctgga gagcgagttcgtgtacggcga ctacaaggtgtacga cgtgcgcaagatgatcgccaagagcgagca ggagatcggcaaggccaccgccaagtacttcttctac a gca a catcatgaa cttcttcaa gaccgagatcaccctggcca acggcgagatccgcaagcgccccctgatcgaga ccaacggcgagaccggcgagatcgtgtgggacaagggccgcgacttcgc caccgtgc gca aggtgctga gcatgccccaggtgaaca tcgtgaaga agaccgaggtgcagaccggcggcttcagcaaggagagcatcctgcccaagcgcaac agcga caagctga tcgcccgcaagaaggactgggac cccaagaagtacggcggcttcgacagccccaccgtggcctacagcgtgctggtggtggccaaggtgga gaagggcaa gagcaaga agctgaagagcgtgaaggagctgctgggcatcaccatcat ggagcgc agca gcttcgagaagaa ccccatcgacttcctggaggcca agggctaca aggaggtga agaaggacctgatcatcaagctgcccaagt aca gcctgttcgagctggagaa cggccgcaagcgcatgctggcc agcgccggcgagctgcagaagggcaacgagctggccctgcccagcaa gtacgtga acttcctgta cctggccagcca ctacgaga agctgaagggcagccccgaggacaacgagcagaagcagctgttcgtg 
gagca gcacaagcactacctggacgaga tcatcgagcagatcagcgagttcagcaagcgcgtgatcctggccgacgccaacctggaca aggtgctga gcgcctacaa caagcaccgcga caagcccatccgc gagca ggccgagaacatcatccacctgttcaccctgacca acctgggcgcccccgccgccttcaagtacttcgacaccaccatcga ccgcaagcgctacaccagca ccaaggaggtgctggacgc caccctgat cca ccagagcatcaccggtctgtacgagacccgcatcgacctga gccagctgggcggcgacggcggctccgga cctccaaa gaaaaagagaaa agtatacccctacgacgtgcccgactacgcctgttaagcg gccgca ctcgagcaccaccaccaccaccactgagatccggctgctaacaaagcccgaaaggaagctgagttggctgctgccaccgctgagcaataactagcataa ccccttggggcctctaaacg gggtttttgctgaaaggaggaactatatccggattggcgaatgggacgcgccctgtagcggcgcattaagcgcggcgggtgtggtggtta cgcgcagcgtga ccgctacacttgccagcgccctagcgcccgct cctttcgctttcttcccttcctttctcgccacgttcgccggctttccccgtcaagctcta aatcgggggctccctttagggttccgatttagtgctttacggcacctcgaccccaaaa aact tga ttagggtgatggttca cgtagtgggccatcgccctgatagacggttttcgccctttga cgttggagtccacgttctttaatagtggactcttgttccaaactggaacaacactcaa ccctatctcggtctattcttttgatttataa gggattttg ccgatttcggcctattggttaaaaaatgagctgatttaacaaaaa

\section{>7 SpdCas9-VirD2-Mutated}

Ccaccctgaattgactctcttccgggcgctatcatgcca taccgcgaaaggttttgcgccattcgatggtgtccgggatct cgacgctctcccttatgcgactcctgcattaggaagcagcccagtagtaggttgag gccgttgagcaccgccgccgcaaggaatggtgcatgcaaggaga tggcgcccaa cagtcccccggcca cggggcctgccaccatacccacgccgaaacaagcgctcatgagcccgaagtggcgagcccgatc ttccccatcggtgatgtcggcga tataggcgccagcaaccgcacctgtggcgccggtga tgccggccacgatgcgtccggcgtagaggatcgagatctcgatcccgcgaaattaatacgactcactatagggga attgtgagcggataacaattcccctctagaaa taattttgttta actttaagaaggaga tataccatgggcagcagccatcatcatcatcatcacagcagcggcctggtgccgcgcggcagccat atggctagcat gactggtgga cagcaaatgggtcgcggatccgaattcgagctccgtcgaca agcttgcggccgcatggacaagaagtacagcatcggcctggCTatcggtaccaacagcgtgggctgggccgtgatcaccgac gagta caaggtgcccagca agaagttcaaggtgctgggcaacaccgaccgccacagca tcaagaagaacctgatcggcgccctgctgttcgacagcggcgagaccgccgaggccacccgcctga agcgcacc gcccgccgccgctaca cccgccgcaagaaccgcatctgctacctgcaggagatcttcagcaacgaga tggccaaggtggacgacagcttcttccaccgcctggaggagagcttcctggtggaggaggacaaga agca cgagcgccaccccatcttcggcaacatcgtggacgaggtggcctaccacgagaagtaccccaccatctaccacctgcgcaagaagctggtggacagca ccgacaaggccgacctgcgcctga tctacct ggccctggcccacatgatcaagttccgcggccacttcctgatcgagggcgacctgaa ccccgacaa cagcgacgtggacaagctgttcatccagctggtgcagacctacaaccagctgttcgagga gaacccca tca a cgccagcggcgtggacgcca aggccatcctgagcgcccgcctgagcaagagccgccgcctggagaacctgatcgcccagctgcc cggcgagaagaagaa cggcctgttcggcaacctgatcgccctga gcctgggcctgacccccaacttca agagcaa cttcgacctggccga ggacgccaa gctgcagctga gcaaggacacctacgacgacgacctgga caacctgctggcccagatcggcga ccagtacgccgacct gttcctggccgccaagaacctgagcgacgccatcctgctgagcga catcctgcgcgtgaacaccgagatcacca aggcccccctgagcgccagcatga tcaagcgcta cgacgagca ccaccaggacctgacc ctgctgaaggccctggtgcgccagcagctgcccgaga agtacaaggagatcttcttcgaccagagcaagaacggctacgccggctacatcga cggcggcgccagccaggaggagttctacaagttcatcaagc ccatcctggaga agatgga cggcaccgaggagctgctggtgaagctgaa ccgcgaggacctgctgcgcaagcagcgcaccttcgacaacggcagcatcccccaccagatccacctgggcgagctgcacgccat cctgcgccgccaggaggacttcta ccccttcctgaaggacaaccgcgagaaga tcgagaagatcctgaccttccgcatcccctactacgtgggccccctggcccgcggcaacagccgcttcgcctggatgacccg caaga gcgaggagaccatcaccccctggaacttcgaggaggtggtggacaagggcgccagcgcccagagcttcatcgagcgcatgaccaacttcgacaagaacctgccca acgagaa ggtgctgc ccaagca cagcctgctgtacgagtacttcaccgtgtacaacgagctgaccaaggtgaagta cgtgaccgagggca tgcgcaagcccgccttcctgagcggcgagcagaagaaggcca tcgtggacctgctgt tcaagacca accgcaaggtgaccgtgaagcagctgaa ggaggactacttcaaga agatcgagtgcttcgaca gcgtggagatcagcggcgtggaggaccgcttca acgccagcctgggcacctac ca cgacctgctgaagat catca aggacaaggacttcctggacaacgaggagaacgaggacatcctggaggacatcgtgctgacc tgaccctgttcgaggaccgcgagatgatcgaggagcgcctgaagacctacgcccacctgttcgac gacaa ggtga tgaagcagctgaagcgccgccgctacaccggctggggccgcctgagccgcaagctta tcaacggcatccgcgacaagcagagcggcaagaccatcctggacttcctgaagagcgacggcttc gccaa ccgcaacttcatgca gctgatcca cgacgacagcctgaccttcaaggaggacatccagaaggcccaggtgagcggccagggcgaca gcctgcacgagcacatcgccaacctggccggcagccc cgcc atca aga agggcatcctgcagaccgtgaaggtggtgga cgagctggtgaaggtgatgggccgccacaa gcccgagaacatcgtgatcgagatggcccgcgagaacca gaccacccaga agggccagaaga a cagccgcgagcgcatgaagcgcatcgaggagggcatcaaggagctgggcagccagatcctga aggagca ccccgtggagaa cacccagctgca gaacgaga agctgta cctgtactacctgcagaa cggc cgcga ca tgtacgtggaccaggagctggaca tcaaccgcctgagcgacta cgacgtggacGCcatcgtgccccagagcttcctgaa ggacgac agca tcgacaa caaggtgctgacccgcagcgacaagaa ccgcggcaagagcgacaacgtgcccagcgaggaggtggtgaagaagatgaagaactactggcgccagctgctgaacgcca agctgatcacccagcgcaagttcgaca acctgacca aggccgagcgcggcg gcctgagcgagctggacaaggccggcttcatcaagcgccagctggtggagacccgcca gatcaccaagcacgtggcccagatcctggacagccgcatgaacaccaagtacgacgagaacgacaagctgatcc gcgaggtgaa ggtgatcaccctgaagagcaagctggtga gcgacttccgcaaggacttccagttctacaaggtgcgcga gatcaacaactaccaccacgcccacgacgcctacctgaacgccgtggtgggcac cgccctga tcaagaagtaccccaagctgga gagcgagttcgtgtacggcga ctacaaggtgtacga cgtgcgcaagat gatcgccaagagcgagcaggagatcggcaaggccaccgccaagtacttcttctac agca a catcatgaa cttcttcaa gaccgagatcaccctggcca acggcgagatccgcaagcgccccctgatcgaga ccaacggcgagaccggcgagatcgtgtgggacaagggccgcgacttcgc caccgtgc gcaa ggtgctga gcatgccccaggtgaaca tcgtgaaga agaccgaggtgcagaccggcggcttcagc aaggagagcatcctgcccaagcgcaacagcga caagctga tcgcccgcaagaaggactgggac cccaa gaagtacggcggcttcgacagccccaccgtggcctacagcgtgctggtggtggccaaggtgga gaagggcaa gagcaaga agctgaagagcgtgaa ggagctgctgggcatcaccatcat ggagcgc agcagcttcgagaagaa ccccatcgacttcctggaggcca agggctaca aggaggtga agaaggacctgatcatcaagctgcccaagtaca gcctgttcgagctggagaacggccgcaagcgcatgctggcc agcgccggcgagctgcagaagggcaacgagctggccctgcccagcaa gtacgtga act tcctgta cctggccagcca ctacgaga agctgaagggcagccccgaggacaacgagcagaagcagctgttcgtg gagcagcacaagcactacctggacgaga tcatcgagcagatcagcgagttcagcaagcgcgtgatcctggccgacgccaacctggaca aggtgctgagcgcctacaa caagcaccgcga caagcccatccgc gagca ggccgagaacatcatccacctgttcaccctgacca acctgggcgcccccgccgccttcaagtacttcgacaccaccat cga ccgcaagcgctacaccagca ccaaggaggtgctggacgc caccctgat ccaccagagcatcaccggtctgtacgagacccgcatcgacctgagccagctgggcggcgacggcggctccgga cctccaaagaaaaagagaaa agtatacccctacgacgtgcccgactacgcctgtggagg cggtggatctggcggtggcggaagtgggggaggtgga agtggtaccatgcccgatcgagctcaagttatcattcgcattgtgccgggaggtggca ccaagacccttcaacaaattatcaatcagt tggagtatct atcccggaagggcaggctggagctgcagcgttcagcccgacatctcgatattcccctgccaccggatcaaatccacgaacttgcccgaagctgggttcaagagactggaacttatga cgaaagtcagccagacg agga a aggcaacaggagttgacca cccatattattgtaagcttccccgccggta caagccaggtagcggctta tgcggcgagccggga gtgggcagccgagatgtttgggtcaggcgcagggggggggccgata ca actatcttacggccttccacatcgatcgcgaccaccca catctgcatgtcgtcgtcaa tcggcgcgaacttttaggacacggctggctgaagata tctcggcgccatccccaactga attacga cgccctgcgc a ta a agatggccgagatttcacttcgtcatggcattgccctcgatgcgagccgacgagcagaacgtggcatcaccgagcggccgatcacttatgcccaatatcggcgccttgagcggga gcaggc tcgccaaat ccgtttcgaagacgcggatttggaacagtcgtcgccgcaaggagatcatccagaattcagccaacctttcgatacatccccatttgaagcatccgcgggcggaccggaggacatgcctcggcccaacaatcggc aga a tgagtcgcaagttcatctccaggagccagctggtgtcagcaa cgaagccggtgtccttgtgcgggttgcattggagacggagcgccttgctcaaccattcgtttccgaaacca ttctcgcggacga catagg gagcggctcttcgcgtgttgccgagggecgtgtggagagcgcaaaccgcactcccgatattcctcgcgcagcaactgaagctgccacgca cacgacacacgaccggcagcggcgtgcaaagcgtc ctcatgatg acga cggagggccgagtggagcaaaacgtgtgacattggaaggcatcgcggttgggccccaggcgaacgccggcgaacaggatggcagtagtggccccttagta cggcaagctggaacgtctcggccatctc ca ccga cggccacgacgcgggccagcaccgcaaccgattcattgtctgctacagcccacctccagcaa cggagaggtgtcctttcaaagcgtccgcgtgaagatga tgatggagaaccgagtgaacgcaa acg cgagaga gatgagcgcagca aggacgggcgtgggggaaataggagataataa gcggccgcactcgagcaccaccaccaccaccactgagatccggctgctaacaaagcccgaaaggaagctga gttggctg ctgcca ccgctga gcaataactagcata accccttggggcctctaaacgggtcttgaggggtttttgctgaaaggaggaactatatccggattggcgaatgggacgcgccctgtagcggcgcat taagcgcggc gggtgtggtggtta cgcgcagcgtgaccgctacacttgccagcgccctagcgcccgctcctttcgctttcttcccttcctttctcgccacgttcgc cggctttccccgtcaagctctaa atcgggggctccctttagggt tccgatttagtgcttta cggcacctcgaccccaaaaa acttgattagggtga tggttcacgtagtgggccatcgccctgatagacggtttttcgccctttgacgttggagtccacgttctttaat agtggactcttgttc ca a a ctggaacaaca ctcaaccctatctcggtcta ttcttttgattta taaggga ttttgccgatttcggcctattggttaa aaaatgagctgatttaaca aaaatttaacgcgaattttaacaaa atattaacgctta caatttaggtggcacttttcggggaa atgtgcgcggaacccctatttgtttatttttcta aatacattcaaatatgtatccgctcatgaattaa ttcttaga aaaactcatcgagcatcaaatgaaactgcaatttatt cata tcaggattatcaataccatatttttgaaaaagccgtttctgtaatga aggagaaaactcaccgaggcagttccataggatggcaaga tcctggtatcggtctgcgattccgactcgtcca acatca atacaa cctattaatttcccctcgtcaaaa ataaggttatcaagtgagaaatcaccatgagtgacga ctgaatccggtgagaatggcaaaagtttatgcatttctttccagacttgttcaacaggccagcc attacgctcgtc atca aa atcactcgcatcaacca aaccgttattcattcgtgattgcgcctgagcgagacgaaatacgcgatcgctgttaaaaggacaattacaaacaggaatcgaatgca accggcgcaggaacactgccagc 
gcatca aca atattttcacctgaatcagga tattcttctaatacctggaatgctgttttcccgggga tcgcagtggtgagtaa ccatgcatcatcaggagtacggata aaatgcttgatggtcggaagaggcataa attccgtca gccagttta gtctgaccatctcatctgtaacatcattggcaacgctacctttgccatgtttca gaaaca actctggcgcatcgggcttccca tacaatcgatagattgtcgcacctgattgcccga catt atcgcgagcccatttatacccatataaatca gcatccatgttggaatttaatcgcggcctagagcaagacgtttcccgttgaatatggctcataacaccccttgtattactgtttatgtaagcagacagttttattgtt catga ccaa aatcccttaacgtga gttttcgttccactgagcgtcagaccccgta gaaaagatcaaa ggatcttcttgagatccttttttctgcgcgtaa tctgctgcttgcaaacaa aaaaac caccgcta ccag cggtggtttgtttgccggatcaagagccacca actcttttccgaaggtaactggcttcagcagagcgcagata ccaaata ctgtccttctagtgtagccgtagttaggccaccacttca agaactctgtagcaccg ccta catacctcgctctgcta atcctgttaccagtggctgctgccagtggcga taagtcgtgtcttaccgggttggactca agacgatagttaccgga taaggcgca gcggtcgggctga acggggggttcgtgca ca ca gcccagcttggagcgaa cgacctaca ccgaactgagataccta cagcgtgagctatgaga aagcgccacgcttcccgaaggga gaaaggcggacaggtatccggtaagcggcagggtcggaacagga gagcgca cgagggagcttccagggggaaacgcctggtatctttatagtcctgtcgggtttcgccacctctgacttgagcgtcgattttgtgatgctcgtcaggggggcggagcctatggaaa aacgcca gcaacg cggccttttta cggttcctggccttttgctggccttttgctcacatgttctttcctgcgttatcccctgattctgtggata accgtatt accgcctttga gtgagctga taccgctcgccgca gccgaacga ccgagcgca gcgagtcagtga gcgaggaa gcggaaga gcgcctgatgcggtattttctccttacgcatctgtgcggtatttcacaccgcaatggtgca ctctcagtacaatctgctctgatgccgcatagttaagccagtatacac tccgctatcgctacgtgactgggtcatggctgcgccccgacacccgcca acacccgctgacgcgccctgacgggcttgtctgctcccggca tccgcttacagacaagctgtgaccgtctccgggagctgcatgtgtc a ga ggttttcaccgtcatcaccgaaacgcgcgaggcagctgcggtaaagctcatcagcgtggtcgtgaagcgattca cagatgtctgcctgttcatccgcgtccagctcgttgagtttctccagaagcgttaatgtc tggcttctga taaagcgggccatgttaagggcggttttttcctgtttggtcactgatgcctccgtgta agggggatttctgttca tgggggtaatgataccga tgaaacgagagagga tgctcacga tacgggttact gatga tga acatgcccggttactggaacgttgtga gggtaaacaactggcggta tggatgcggcgggaccagaga aaaatcactcagggtcaatgccagcgcttcgttaataca gatgtaggtgttccacaggg tagcca gcagcatcctgcgatgcagatccggaacata atggtgcagggcgctgacttccgcgtttccaga ctttacga aacacggaaaccgaa gaccattca tgttgttgctcaggtcgcagacgttttgcagca gcagtcgcttcacgttcgctcgcgtatcggtgattcattctgctaaccagtaaggcaaccccgccagcctagccgggtcctcaacgacaggagcacgatcatgcgcacccgtggggccgccatgccggcgataat ggcctgcttctcgccgaaacgtttggtggcgggaccagtgacgaa ggcttgagcgagggcgtgcaagattccgaa taccgcaagcgacaggccga tcatcgtcgcgctccagcgaa agcggtcctcgccgaaa atga cccagagcgctgccggcacctgtcctacgagttgcatgataaagaagacagtcataagtgcggcgacgatagtcatgcccecgcccaccggaa ggagctgactgggttgaaggctctcaagggcatcg gtcga ga tcccggtgcctaatgagtgagctaactta cattaattgcgttgcgctcactgcccgctttccagtcgggaaacctgtcgtgccagctgcattaatga atcggccaacgcgcggggagaggcggtttgcg tattgggcgcca gggtggtttttctttca ccagtgaga cgggcaaca gctgattgcccttcaccgcctggccctgagagagttgcagcaagcggtccacgctggtttgccccagca ggcgaaaa tcctgtttgatg gtggtta a cggcgggatataacatgagctgtcttcggtatcgtcgtatcccactaccgagatatccgcaccaa cgcgcagcccggactcggtaatggcgcgcattgcgcccagcgccatctga tc gttggcaacca gcatcgcagtgggaacgatgccctcattcagcatttgcatggtttgttgaaaaccggacatggcactccagtcgccttcccgttccgcta tcggctgaatttgattgcgagtgaga tatttatgccagccagccaga cgca ga cgcgccga gacagaa cttaatgggcccgctaaca gcgcgatttgctggtgacccaatgcgacca gatgctccacgcccagtcgcgtaccgtcttcatggga gaaaataatactgttgat gggtgtctgg tca ga gacatcaaga aataa cgccggaacattagtgca ggcagcttccacagcaatggcatcctggtcatccagcggatagttaa tgatcagcccactgacgcgttgcgcgagaagattgtgca ccgccgctta caggcttcgacgccgcttcgttctaccatcgacaccaccacgctggcaccagttgatcggcgcgagatttaatcgccgcgacaatttgcgacggcgcgtgcagggccagactggaggtggcaacgccaa tcag ca a cgactgtttgcccgccagttgttgtgccacgcggttgggaa tgtaattcagctccgccatcgccgcttccactttttcccgcgttttcgcagaaacgtggctggcctggttcaccacgcgggaaa cggtctgata aga ga caccggcatactctgcgacatcgtataacgttactggtttcacattca 